Homology, Homotopy and Applications, vol.19(1), 2017, pp.293-318

\title{
THE DECOMPOSABILITY OF A SMASH PRODUCT OF $\mathbf{A}_{n}^{2}$-COMPLEXES
}

\author{
ZHONGJIAN ZHU AND JIANZHONG PAN
}

(communicated by John R. Klein)

\begin{abstract}
In this paper, we determine the decomposability of the smash product of two indecomposable $\mathbf{A}_{n}^{2}$-complexes, i.e., $(n-1)$ connected finite $\mathrm{CW}$-complexes with dimension at most $n+2$ $(n \geqslant 3)$.
\end{abstract}

\section{Introduction}

For a finite CW-complex $X$ with co-H-space structure, if $X \simeq X_{1} \vee X_{2}$ with noncontractible $X_{1}$ and $X_{2}$, then $X$ is called decomposable; otherwise $X$ is called indecomposable. One of the basic problems in homotopy theory is to classify indecomposable homotopy types. Although it is impossible to find all indecomposable homotopy types, it is indeed possible to solve this problem in special situations. Let $\mathbf{A}_{n}^{k}$ $(n \geqslant k+1)$ be the homotopy category consisting of $(n-1)$-connected finite CWcomplexes with dimension at most $n+k$. Any complex in $\mathbf{A}_{n}^{k}$ is a suspension and thus a co-H-space. Let $\mathbf{F}_{n}^{k}$ be the full subcategory of $\mathbf{A}_{n}^{k}$ consisting of complexes with torsion-free homology groups, $\mathbf{F}_{n(2)}^{k}$ the full subcategory of $\mathbf{A}_{n}^{k}$ consisting of complexes with 2-torsion-free homology groups, and $\mathbf{F}_{n(2,3)}^{k}$ the full subcategory of $\mathbf{A}_{n}^{k}$ consisting of complexes with 2- and 3-torsion-free homology groups.

In 1950, Chang classified the indecomposable homotopy types in $\mathbf{A}_{n}^{2}(n \geqslant 3)$ [6], that is

(i) Spheres: $S^{n}, S^{n+1}, S^{n+2}$;

(ii) Elementary Moore spaces: $M_{p^{r}}^{n}, M_{p^{r}}^{n+1}$ where $p$ is a prime, $r \in \mathbb{Z}^{+}$and $M_{p^{r}}^{k}$ denotes $M\left(\mathbb{Z} / p^{r}, k\right)$;

(iii) Elementary Chang complexes: $C_{\eta}^{n+2}, C^{(n+2), s}, C_{r}^{(n+2)}, C_{r}^{(n+2), s}$ (see Section 2.1) where $r, s \in \mathbb{Z}^{+}$, where $\mathbb{Z}^{+}$denotes the set of positive integers.

A classification of indecomposable homotopy types of $\mathbf{A}_{n}^{3}(n \geqslant 4)$ was given by Baues and Hennes in [5] and, for $k \geqslant 4$, the classification of indecomposable homotopy types of $\mathbf{A}_{n}^{k}(n \geqslant k+1)$ is wild in the sense similar to that in the representation theory of finite dimensional algebras. Classifications of indecomposable homotopy types of $\mathbf{F}_{n}^{k}(n \geqslant k+1)$ for $k=4,5,6$ are given by Baues and Drozd in $[\mathbf{3}, \mathbf{4}, \mathbf{8}]$ and it is wild for $k \geqslant 7$. Based on these earlier results, in our previous papers [11, 12], we

Received June 14, 2016, revised August 26, 2016; published on April 26, 2017.

2010 Mathematics Subject Classification: 55P10, 55P15.

Key words and phrases: indecomposable, smash product, $\mathbf{A}_{n}^{k}$-complex, cofibre sequence.

Article available at http://dx.doi.org/10.4310/HHA.2017.v19.n1.a15

Copyright (C) 2017, International Press. Permission to copy for private use granted. 
classify indecomposable homotopy types of $\mathbf{F}_{n(2,3)}^{k}(n \geqslant k+1)$ for $k \leqslant 6$ and $\mathbf{F}_{n(2)}^{4}$ $(n \geqslant 5)$.

As pointed out by $\mathrm{Wu}[\mathbf{1 8}]$, starting from an explicit space $X$, one obtains more indecomposable spaces from the self-smash products of $X$ since self-smash products of co-H-spaces admit decompositions. This motivates us to consider the decomposability of smash products of different indecomposable complexes. There are only a few results for this problem. The decomposability of $M\left(\mathbb{Z} / p^{r}, n\right) \wedge M\left(\mathbb{Z} / p^{s}, n\right)$ is well known $[\mathbf{9}]$. $\mathrm{Wu}[\mathbf{1 6}]$ proved that $M(\mathbb{Z} / 2, n) \wedge C_{\eta}^{n+2}$ and $C_{\eta}^{n+2} \wedge C_{\eta}^{n+2}$ are indecomposable. As a main result in this paper, we determine the decomposability of all remaining smash products of two indecomposable complexes in $\mathbf{A}_{n}^{2}(n \geqslant 3)$ and give the decomposition whenever possible. Since the suspension functor $\Sigma: \mathbf{A}_{n}^{2} \rightarrow \mathbf{A}_{n+1}^{2}$ is an equivalence for $n \geqslant 3$, it suffices to deal with the case $n=3$.

Theorem 1.1 (Main theorem). For $r, s, r^{\prime}, s^{\prime}, u \in \mathbb{Z}^{+}$,

- $M_{2^{u}}^{3} \wedge C_{\eta}^{5}, C_{r}^{5} \wedge C^{5, s}, C_{r}^{5} \wedge C_{r^{\prime}}^{5}, C^{5, s} \wedge C^{5, s^{\prime}}, C_{\eta}^{5} \wedge C_{r}^{5}, C_{\eta}^{5} \wedge C^{5, s}, C_{\eta}^{5} \wedge C_{r}^{5, s}$ are indecomposable;

- $M_{2^{u}}^{3} \wedge C_{r}^{5}$ and $M_{2^{u}}^{3} \wedge C^{5, r}$ are

$\diamond$ indecomposable for $u>r$;

$\diamond$ homotopy equivalent to $M_{2^{u}}^{3} \wedge C_{\eta}^{5} \vee M_{2^{u}}^{7}$ for $r \geqslant u$;

- $M_{2^{u}}^{3} \wedge C_{r}^{5, s}$ is homotopy equivalent to

$\diamond C_{r}^{8, s} \vee C_{r}^{9, s}$ for $u>r, s$;

$\diamond M_{2^{u}}^{3} \wedge C_{r}^{5} \vee M_{2^{u}}^{7}$ for $r<u \leqslant s ;$

$\diamond M_{2^{u}}^{3} \wedge C^{5, s} \vee M_{2^{u}}^{7}$ for $s<u \leqslant r$;

$\diamond M_{2^{u}}^{3} \wedge C_{\eta}^{5} \vee M_{2^{u}}^{7} \vee M_{2^{u}}^{7}$ for $u \leqslant r$ and $u \leqslant s ;$

- $C_{u}^{5} \wedge C_{r}^{5, s}$ is

$\diamond$ homotopy equivalent to $C_{r}^{9, s} \vee C_{\eta}^{5} \wedge C_{r}^{5, s}$ for $u \geqslant r$ and $u \geqslant s$;

$\diamond$ homotopy equivalent to $C_{s}^{9, r} \vee C_{\eta}^{5} \wedge C_{s}^{5, s}$ for $u=s<r$;

$\diamond$ indecomposable, otherwise;

- $C^{5, u} \wedge C_{r}^{5, s}$ is

$\diamond$ homotopy equivalent to $C_{r}^{9, s} \vee C_{\eta}^{5} \wedge C_{r}^{5, s}$ for $u \geqslant r$ and $u \geqslant s$;

$\diamond$ homotopy equivalent to $C_{s}^{9, r} \vee C_{\eta}^{5} \wedge C_{r}^{5, r}$ for $u=r<s$;

$\diamond$ indecomposable, otherwise;

- $C_{r}^{5, s} \wedge C_{r^{\prime}}^{5, s^{\prime}}$

$\diamond$ if $s \geqslant r, r^{\prime}, s^{\prime}$

$$
C_{r}^{5, s} \wedge C_{r^{\prime}}^{5, s^{\prime}} \simeq \begin{cases}C_{r}^{9, s} \vee C_{r}^{9, s} \vee C_{\eta}^{5} \wedge C_{r}^{5, s}, & s=s^{\prime}=r^{\prime}>r ; \\ C_{r}^{9, s} \vee C_{r^{\prime}}^{5} \wedge C_{r}^{5, s}, & s=s^{\prime}>r^{\prime}>r ; \\ C_{r}^{9, s} \vee C^{5, s^{\prime}} \wedge C_{r}^{5, s}, & s=r^{\prime}>s^{\prime}>r ; \\ C_{r^{\prime}, s^{\prime}}^{9,} \vee C_{r^{\prime}}^{9, s^{\prime}} \vee C_{\eta}^{5} \wedge C_{r^{\prime}}^{5, s^{\prime}}, & s \geqslant r \geqslant r^{\prime}, s^{\prime} ; \\ C_{r^{\prime}, s^{\prime}} \vee C_{s^{\prime}}^{9, r^{\prime}} \vee C_{\eta}^{5} \wedge C_{r}^{5, r}, & s \geqslant r^{\prime}>s^{\prime}=r ; \\ C_{r^{\prime}}^{9, s^{\prime}} \vee C_{r}^{5} \wedge C_{r^{\prime}}^{5, s^{\prime}}, & \text { otherwise; }\end{cases}
$$

$\diamond$ if $r \geqslant r^{\prime}, s^{\prime}$ and $r>s$ 


$$
C_{r}^{5, s} \wedge C_{r^{\prime}}^{5, s^{\prime}} \simeq \begin{cases}C_{r}^{9, s} \vee C_{r}^{9, s} \vee C_{\eta}^{5} \wedge C_{r}^{5, s}, & r=r^{\prime}=s^{\prime}>s ; \\ C_{r}^{9, s} \vee C^{5, s^{\prime}} \wedge C_{r}^{5, s}, & r=r^{\prime}>s^{\prime}>s ; \\ C_{r}^{9, s} \vee C_{r^{\prime}}^{5, \wedge} C_{r}^{5, s}, & r=s^{\prime}>r^{\prime}>s ; \\ C_{r^{\prime}, s^{\prime}}^{9,} \vee C_{r^{\prime}}^{9, s^{\prime}} \vee C_{\eta}^{5} \wedge C_{r^{\prime}}^{5, s^{\prime}}, & r>s \geqslant r^{\prime}, s^{\prime} ; \\ C_{r^{\prime}, s^{\prime}}^{9} \vee C_{s^{\prime}}^{9, r^{\prime}} \vee C_{\eta}^{5} \wedge C_{s}^{5, s}, & r \geqslant s^{\prime}>r^{\prime}=s ; \\ C_{r^{\prime}}^{9, s^{\prime}} \vee C^{5, s} \wedge C_{r^{\prime}}^{5, s^{\prime}}, & \text { otherwise. }\end{cases}
$$

Remark 1.2. For $M_{p^{r}}^{3}$, prime $p \neq 2$, it is easy to check that $M_{p^{r}}^{3} \wedge C_{\eta}^{5} \simeq M_{p^{r}}^{6} \vee M_{p^{r}}^{8}$; $M_{p^{r}}^{3} \wedge C_{r^{\prime}}^{5} \simeq M_{p^{r}}^{8} ; M_{p^{r}}^{3} \wedge C^{5, s} \simeq M_{p^{r}}^{6} ; M_{p^{r}}^{3} \wedge C_{r}^{5, s} \simeq * ; M_{p^{r}}^{3} \wedge M_{q^{r}}^{3} \simeq *$ for prime $q \neq$ $p$ (* is the point space). We will not discuss these cases any more in the following.

It is known from Theorem 1.2 of [14] that for any $p$-local CW-complex, there is a functorial decomposition $\Omega \Sigma X \simeq A^{\min }(X) \times \Omega\left(\bigvee_{n=2}^{\infty} Q_{n}^{\max }(X)\right)$ which is useful to calculate homotopy groups of $\Sigma X . Q_{n}^{\max }(X)$ is a wedge summand of $\Sigma X^{(n)}$, where $X^{(n)}$ is an $n$-fold self-smash product of $X$. In order to determine the homotopy type of $Q_{n}^{\max }(X)$, it is significant to decompose $X^{(n)}$ to a wedge of indecomposable spaces. The decomposition of self-smash products is easy for $M_{p^{r}}^{n}(p>2)$ and $M_{2^{s}}^{n}(s>1)$. The decomposition of self-smash products is obtained by Wu [16] for $M_{2}^{n}$ and $C_{\eta}^{n}$. In a sequel we will study the decomposition of self-smash products for Chang complexes.

Section 2 contains necessary notation and lemmas. Related results of elementary Moore spaces and Chang-complexes are stated in Section 3. In Section 5, we prove the last part of Theorem 1.1 by determining the decomposition of $C_{r}^{5, s} \wedge C_{r^{\prime}}^{5, s^{\prime}}$ while the proof of other cases in Theorem 1.1 is given in Section 4.

\section{Preliminaries}

\subsection{Some notation}

- All spaces are suspensions of simply connected finite CW-complexes.

- $|G|$ denotes the order of a group $G$ and $|g|$ denotes the order of an element $g$ in group $G$. If $G$ is an abelian group with decomposition $G \cong C_{1} \oplus C_{2} \oplus \cdots \oplus C_{m}$, where $C_{t}$ is a cyclic group with order infinity or a power of a prime for $t=$ $1, \cdots, m$, then define $\operatorname{dim} G:=m$.

- If $X$ is a subspace of $L, Y \simeq L / X$, then $i$ denotes the canonical inclusion $X \hookrightarrow L$, $q$ denotes the canonical projection $L \rightarrow Y$. Especially for Moore spaces $M_{2^{k}}^{n}$, sometimes we denote $i: S^{n} \hookrightarrow M_{2^{k}}^{n}$ by $i_{n}$ and $q: M_{2^{k}}^{n} \rightarrow S^{n+1}$ by $q_{n}$.

- Denote by $H_{*} X:=H_{*}(X ; \mathbb{Z})$ and $H^{*}(X ; \mathbb{Z} / 2)$ the reduced homology groups and cohomology groups of the space $X$, respectively.

- Let $\mathbf{C}_{f}$ be the mapping cone of a map $f$. Denote by $\left[\mathbf{C}_{f}, \mathbf{C}_{f^{\prime}}\right]_{\beta}^{\alpha}$ the set of homotopy classes of maps $h$ which satisfy the following homotopy commutative diagram:

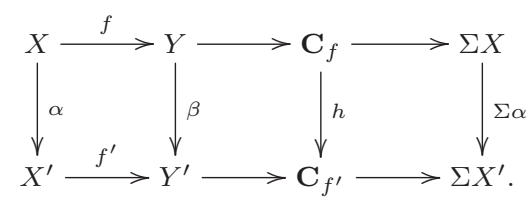


- For $X_{i}$ and $Y_{j}(i=1, \cdots, t, j=1, \cdots, s)$ objects in an additive category (such as $\mathbf{A}_{n}^{k}(k \geqslant n+2)$ or the category of abelian groups), we denote by $f:=\left(f_{i j}\right)=$ $\left(\begin{array}{lll}f_{11} & \cdots & f_{1 t} \\ \cdots & \cdots & \cdots \\ f_{s 1} & \cdots & f_{s t}\end{array}\right): \bigoplus_{i=1}^{t} X_{i} \rightarrow \bigoplus_{j=1}^{s} Y_{j}$ a morphism such that $p_{Y_{i}} f j_{X_{j}}=$ $f_{i j}$, where $j_{X_{j}}$ and $p_{Y_{i}}$ are canonical inclusions and projections, respectively. Sometimes, $\left(f_{i j}\right)$ is written graphically as follows to indicate the domain and codomain:

\begin{tabular}{c|cccc}
\multicolumn{1}{c}{} & \multicolumn{1}{c}{$X_{1}$} & $X_{2}$ & $\cdots$ & $X_{t}$ \\
\cline { 2 - 5 }$Y_{1}$ & $f_{11}$ & $f_{12}$ & $\cdots$ & $f_{1 t}$ \\
$Y_{2}$ & $f_{21}$ & $f_{22}$ & $\cdots$ & $f_{2 t}$ \\
$\vdots$ & $\vdots$ & $\vdots$ & $\vdots$ & $\vdots$ \\
$Y_{s}$ & $f_{s 1}$ & $f_{s 2}$ & $\cdots$ & $f_{s t}$ \\
\cline { 2 - 5 } & & &
\end{tabular}

To simplify the text, we fix names for the following elementary transformations:

(i) $-\mathbf{r}_{n}\left(-\mathbf{c}_{n}\right)$ : composing $n$-th row (column) with $-i d$;

(ii) $\mathbf{c}_{m} f+\mathbf{c}_{n}$ : adding the $m$-th column, composed with map $f$, to the $n$-th column;

(iii) $g \mathbf{r}_{m}+\mathbf{r}_{n}$ : adding the $m$-th row, composed with map $g$, to the $n$-th row;

(iv) $k \mathbf{r}_{m}+\mathbf{r}_{n}\left(k \mathbf{c}_{m}+\mathbf{c}_{n}\right)$ : adding $k$ times the $m$-th row (column) to the $n$-th row (column), where $k \in \mathbb{Z}^{+}$.

- In the rest of the paper, $S_{\omega}^{k}=S^{k}$ for $\omega \in\{1,2,3,4, a, b\}$.

- $\eta=\eta_{n} \in\left[S^{n+1}, S^{n}\right]$ is a Hopf map for $n \geqslant 3$ and $\varrho=\varrho_{n} \in\left[S^{n+3}, S^{n}\right] \cong \mathbb{Z} / 24$ is a fixed generator for $n \geqslant 5$.

- $\kappa, \kappa^{\prime}, \varepsilon, \varepsilon^{\prime} \in\{0,1\}$.

- $k$ times the identity map $i d$ of $\Sigma X$ is written as $k: \Sigma X \rightarrow \Sigma X$ for nonzero integer $k$ (hence, $1=i d$ ).

- For $k \geqslant 5$, and $r, s \in \mathbb{Z}^{+}$, let

$$
\begin{aligned}
& C^{k, s}=\left(S^{k-2} \vee S^{k-1}\right) \bigcup_{\left(\begin{array}{c}
\eta \\
2^{s}
\end{array}\right)} \mathbf{C} S^{k-1} ; \quad C_{r}^{k}=S^{k-2} \bigcup_{\left(2^{r}, \eta\right)} \mathbf{C}\left(S^{k-2} \vee S^{k-1}\right) ; \\
& C_{r}^{k, s}=\left(S^{k-2} \vee S^{k-1}\right) \bigcup_{\left(\begin{array}{c}
2^{r}, \eta \\
0,2^{s}
\end{array}\right)} \mathbf{C}\left(S^{k-2} \vee S^{k-1}\right) ; \quad C_{\eta}^{k}=S^{k-2} \bigcup_{\eta} \mathbf{C} S^{k-1} .
\end{aligned}
$$

\subsection{Spanier-Whitehead duality}

If $\mathbf{A}_{n}^{k}$ is in the stable range, i.e., $[X, Y] \stackrel{\Sigma^{m}}{\longrightarrow}\left[\Sigma^{m} X, \Sigma^{m} Y\right]$ is isomorphic for any $X, Y \in \mathbf{A}_{n}^{k}$ and any $m \in \mathbb{Z}^{+}$, then there is a contravariant isomorphism of additive categories

$$
D=D_{2 n+k}: \mathbf{A}_{n}^{k} \rightarrow \mathbf{A}_{n}^{k},
$$

which is called Spanier-Whitehead duality (or $(2 n+k)$-duality).

$D$ satisfies the following properties from $[\mathbf{2}, \mathbf{7}, \mathbf{1 3}]$ :

\section{Proposition 2.1.}

(i) $D^{2}$ is equal to the identity functor;

(ii) $[X, Y] \stackrel{D(\cong)}{\longrightarrow}[D Y, D X] \cong\left[D Y \wedge X, S^{2 n+k}\right]$;

(iii) $\left[S^{n+q}, D X\right] \cong\left[X, S^{n+k-q}\right]$ and $\left[S^{n+q}, X\right] \cong\left[D X, S^{n+k-q}\right]$ for $n \leqslant q \leqslant n+k$; 
(iv) $D(X \vee Y) \simeq D X \vee D Y ; D(X \wedge Y) \simeq D X \wedge D Y$, that is for $X \in \mathbf{A}_{n}^{k}, Y \in \mathbf{A}_{m}^{l}$, then $X \wedge Y \in \mathbf{A}_{n+m}^{k+l}$ and $D_{2(n+m)+k+l}(X \wedge Y) \simeq D_{2 n+k} X \wedge D_{2 m+l} Y$.

(vi) Let $\{X, Y\}:=\lim _{m \rightarrow+\infty}\left[\Sigma^{m} X, \Sigma^{m} Y\right]$. Then for any $C W$-complex $Z$,

$$
\{X \wedge Y, Z\} \cong\left\{X \wedge S^{2 n+k}, D Y \wedge Z\right\}
$$

Note: It follows from (i) and (iv) above that $X$ is indecomposable if and only if $D X$ is indecomposable.

Example 2.2. (Page 49 of [1]) For the Spanier-Whitehead duality $D: \mathbf{A}_{n}^{2} \rightarrow \mathbf{A}_{n}^{2}$ $(n \geqslant 3)$, we have $D S^{n}=S^{n+2}, D S^{n+1}=S^{n+1}, D M_{p^{r}}^{n}=M_{p^{r}}^{n+1}, D C_{\eta}^{n+2}=C_{\eta}^{n+2}$, $D C_{r}^{(n+2), s}=C_{s}^{(n+2), r}, D C_{r}^{n+2}=C^{(n+2), r}$.

\subsection{Some lemmas}

Lemma 2.3. ([च]) For a cofibre sequence $X \stackrel{f}{\longrightarrow} Y \stackrel{i}{\longrightarrow} \mathbf{C}_{f}$,

$$
X \wedge Z \stackrel{f \wedge i d}{\longrightarrow} Y \wedge Z \stackrel{i \wedge i d}{\longrightarrow} \mathbf{C}_{f} \wedge Z
$$

is also a cofibre sequence. That is $\mathbf{C}_{f \wedge i d} \simeq \mathbf{C}_{f} \wedge Z$.

Lemma 2.4. (Lemma 14.30 of [13]) For

$$
X \stackrel{f}{\longrightarrow} U \stackrel{i}{\longrightarrow} \mathbf{C}_{f}, \quad Y \stackrel{g}{\longrightarrow} V \stackrel{i}{\longrightarrow} \mathbf{C}_{g},
$$

$\mathbf{C}_{f} \wedge \mathbf{C}_{g}=(U \wedge V) \bigcup_{\mu} \mathbf{C}(X \wedge V \vee U \wedge Y) \bigcup_{\nu} \mathbf{C C}(X \wedge Y)$ where $\mu=(f \wedge i d, i d \wedge g)$ and $\left(\mathbf{C}_{f} \wedge \mathbf{C}_{g}\right) /(U \wedge V) \simeq(\Sigma(X \wedge V) \vee \Sigma(U \wedge Y)) \bigcup_{\nu^{\prime}} \mathbf{C} \Sigma(X \wedge Y)$, where

$$
\nu^{\prime}=\left(\begin{array}{c}
\Sigma i d \wedge g \\
-\Sigma f \wedge i d
\end{array}\right)
$$

Lemma 2.5. Let $A \in \mathbf{A}_{6}^{4}$, with homology groups of the form $\mathbb{Z}^{r} \oplus \mathbb{Z} / 2^{r_{1}} \oplus \cdots \oplus \mathbb{Z} / 2^{r_{\varsigma}}$ for some non-negative integers $r, r_{1}, \cdots, r_{\varsigma}$. Suppose that

(i) $\operatorname{dim} H_{9} A+\operatorname{dim} H_{10} A=1$ and $H^{6}(A ; \mathbb{Z} / 2) \cong H^{10}(A ; \mathbb{Z} / 2) \cong \mathbb{Z} / 2$ with generators $a_{6}$ and $a_{10}$, respectively, satisfying $S q^{4} a_{6}=a_{10}$;

(ii) $\operatorname{dim} H^{8}(A ; \mathbb{Z} / 2) \geqslant 2$ and there are nonzero elements $a_{8} \neq a_{8}^{\prime} \in H^{8}(A ; \mathbb{Z} / 2)$ such that $S q^{2} a_{8}=S q^{2} a_{8}^{\prime}=a_{10}$;

(iii) $S q^{2} a_{6}=a_{8}+a_{8}^{\prime}+a_{8}^{\prime \prime} \neq 0$ for some $a_{8}^{\prime \prime} \in H^{8}(A ; \mathbb{Z} / 2)$ such that $S q^{2} a_{8}^{\prime \prime}=0$.

If $A \simeq X \vee Y$ and $H_{6} X \neq 0$, then $H_{t} X \cong H_{t} A$ for $t=6,9,10$ and $\operatorname{dim} H^{8}(X ; \mathbb{Z} / 2) \geqslant$ 2, hence $\operatorname{dim} H_{7} X+\operatorname{dim} H_{8} X \geqslant 2$.

Proof. We have

$$
H^{*}(X ; \mathbb{Z} / 2) \underset{j_{1}^{*}}{\stackrel{p_{1}^{*}}{\rightleftarrows}} H^{*}(A ; \mathbb{Z} / 2) \underset{j_{2}^{*}}{\stackrel{p_{2}^{*}}{\leftrightarrows}} H^{*}(Y ; \mathbb{Z} / 2),
$$

where $j_{1}, j_{2}, p_{1}, p_{2}$ are the canonical inclusions and projections and $j_{u}^{*} p_{u}^{*}=i d$ which implies that $p_{u}^{*}$ is injective and $j_{u}^{*}$ is surjective for $u=1,2$.

Since $H_{6} X \neq 0$, we get that $H_{6} X \cong H_{6} A, H_{6} Y=0$ and $H^{6}\left(p_{1} ; \mathbb{Z} / 2\right)$ is isomorphic, hence there is $0 \neq x_{6} \in H^{6}(X ; \mathbb{Z} / 2)$ such that $p_{1}^{*}\left(x_{6}\right)=a_{6}$. It follows from 
$p_{1}^{*}\left(S q^{4} x_{6}\right)=S q^{4} p_{1}^{*}\left(x_{6}\right)=a_{10} \neq 0$ that $0 \neq S q^{4} x_{6} \in H^{10}(X ; \mathbb{Z} / 2)$ which implies $H_{9} X$ $\cong H_{9} A$ and $H_{10} X \cong H_{10} A$ by (i).

By $j_{1}^{*}\left(a_{10}\right)=j_{1}^{*}\left(p_{1}^{*}\left(S q^{4} x_{6}\right)\right)=S q^{4} x_{6} \neq 0$ and $S q^{2} j_{1}^{*}\left(a_{8}\right)=S q^{2} j_{1}^{*}\left(a_{8}^{\prime}\right)=j_{1}^{*}\left(a_{10}\right)$, we get $j_{1}^{*}\left(a_{8}\right) \neq 0$ and $j_{1}^{*}\left(a_{8}^{\prime}\right) \neq 0$ in $H^{8}(X ; \mathbb{Z} / 2)$.

Since $S q^{2} a_{6}=a_{8}+a_{8}^{\prime}+a_{8}^{\prime \prime} \neq 0 \quad$ and $S q^{2} a_{8}^{\prime \prime}=0, \quad p_{1}^{*}\left(S q^{2} x_{6}\right)=S q^{2}\left(a_{6}\right)=a_{8}+$ $a_{8}^{\prime}+a_{8}^{\prime \prime} \neq 0$ and $p_{1}^{*} S q^{2} S q^{2} x_{6}=2 a_{10}=0$, thus $S q^{2} x_{6} \neq 0$ and $S q^{2} S q^{2} x_{6}=0$. But $S q^{2}\left(j_{1}^{*}\left(a_{8}\right)\right)=j_{1}^{*}\left(a_{10}\right) \neq 0$, we have $j_{1}^{*}\left(a_{8}\right) \neq S q^{2} x_{6}$, thus $\operatorname{dim} H^{8}(X ; \mathbb{Z} / 2) \geqslant 2$.

It follows from $H^{8}(X ; \mathbb{Z} / 2)=\operatorname{Hom}\left(H_{8} X, \mathbb{Z} / 2\right) \oplus \operatorname{Ext}\left(H_{7} X, \mathbb{Z} / 2\right)$ that $\operatorname{dim} H_{7} X+$ $\operatorname{dim} H_{8} X \geqslant 2$.

A complex $X$ is called 2-local if all homotopy groups, or equivalently all homology groups, of $X$ are finitely generated $\mathbb{Z}_{(2)}$-modules, where $\mathbb{Z}_{(2)}$ is the 2-localization of $\mathbb{Z}$. Let $X_{(2)}$ be the 2-localization of $X$ and denote by $X \simeq_{(2)} Y$ if $X_{(2)} \simeq Y_{(2)}$.

\section{Lemma 2.6.}

(i) Let $X_{1}=S^{m} \cup_{f_{1}} \mathbf{C} X_{1}^{\prime}, X_{2}=S^{m} \cup_{f_{2}} \mathbf{C} X_{2}^{\prime}$ be two (resp. 2-local) complexes, where $X_{1}^{\prime}$ and $X_{2}^{\prime}$ are $m$-connected. If $X_{1} \simeq X_{2}$ and $H_{m} X_{1}=\mathbb{Z} / 2^{s}$ for some $s \in$ $\mathbb{Z}^{+}$, then $X_{1} / S^{m} \simeq_{(2)} X_{2} / S^{m} \quad$ (resp. $\left.X_{1} / S^{m} \simeq X_{2} / S^{m}\right)$, i.e., $\Sigma X_{1}^{\prime} \simeq_{(2)} \Sigma X_{2}^{\prime}$ (resp. $\left.\Sigma X_{1}^{\prime} \simeq \Sigma X_{2}^{\prime}\right)$.

(ii) Let $X_{1}=X_{1}^{(n-1)} \cup_{g_{1}} \mathbf{C} S^{n-1}, X_{2}=X_{2}^{(n-1)} \cup_{g_{2}} \mathbf{C} S^{n-1}$ be two (resp. 2-local) complexes, where $X_{1}^{(n-1)}$ and $X_{2}^{(n-1)}$ are $(n-1)$-skeleton of $X_{1}$ and $X_{2}$, respectively. If $X_{1} \simeq X_{2}$ and $\left[X_{1}, S^{n}\right]=\mathbb{Z} / 2^{t}$ for some $t \in \mathbb{Z}^{+}$, then $X_{1}^{(n-1)} \simeq{ }_{(2)} X_{2}^{(n-1)}$ $\left(\right.$ resp. $\left.X_{1}^{(n-1)} \simeq X_{2}^{(n-1)}\right)$.

Proof. It suffices to prove when $X_{1}$ and $X_{2}$ are 2-local.

We only prove (i), since the proof of (ii) is dual to the proof of (i).

There are cofibre sequences

$$
X_{j}^{\prime} \stackrel{f_{j}}{\longrightarrow} S^{m} \stackrel{i_{j}}{\longrightarrow} X_{j} \stackrel{q_{j}}{\longrightarrow} \Sigma X_{j}^{\prime} \stackrel{\Sigma f_{j}}{\longrightarrow} S^{m+1}, \quad j=1,2 .
$$

Given a homotopy equivalence $\alpha: X_{1} \stackrel{\simeq}{\longrightarrow} X_{2}$, it induces

$$
\alpha_{*}: \pi_{m} X_{1} \stackrel{\cong}{\longrightarrow} \pi_{m} X_{2} \cong \mathbb{Z} / 2^{s} .
$$

Since $i_{j}$ is a generator of $\pi_{m} X_{j}$ for $j=1,2$, there is an odd integer $k$ such that $\alpha_{*} k i_{1}=i_{2} \in \pi_{m} X_{2}$. Since $X_{1}$ and $X_{2}$ are 2-local, $k \alpha: X_{1} \rightarrow X_{2}$ is also a homotopy equivalence. Take $\alpha^{\prime} \in\left[\Sigma X_{1}^{\prime}, \Sigma X_{2}^{\prime}\right]_{k \alpha}^{i d}$, then we have $\alpha^{\prime}: \Sigma X_{1}^{\prime} \stackrel{\simeq}{\longrightarrow} \Sigma X_{2}^{\prime}$.

\section{Moore spaces and Chang-complexes}

In this section, we will collect some basic facts about Chang-complexes and Moore spaces.

\subsection{Moore spaces}

We list some results of maps between Moore spaces from [5].

$$
\left[M_{2^{r}}^{n}, M_{2^{t}}^{n}\right]=\left\{\begin{array}{ll}
\mathbb{Z} / 4\langle B(\chi)\rangle, & r=t=1 ; \\
\mathbb{Z} / 2^{\min (r, t)}\langle B(\chi)\rangle \oplus \mathbb{Z} / 2\langle i \eta q\rangle, & \text { otherwise, }
\end{array} \quad(n \geqslant 3)\right.
$$

where $B(\chi)$ is given by Proposition 2.3 of [5], which satisfies 


$$
H_{n} B(\chi)=\chi: \mathbb{Z} / 2^{r} \rightarrow \mathbb{Z} / 2^{t}, \quad \chi(1)=1 .
$$

$B(\chi) \in\left[M_{2^{r}}^{n}, M_{2^{t}}^{n}\right]_{i d}^{2^{r-t}}$ for $r \geqslant t$ and $B(\chi) \in\left[M_{2^{r}}^{n}, M_{2^{t}}^{n}\right]_{2^{r-t}}^{i d}$ for $r \leqslant t$; If $r=t$, then $B(\chi)=i d$ and if $r=t=1$, then $i \eta q=2 B(\chi)=2$.

$$
\begin{aligned}
& {\left[S^{n+1}, M_{2^{t}}^{n}\right]=\mathbb{Z} / 2\langle i \eta\rangle, \quad\left[M_{2^{t}}^{n}, S^{n}\right]=\mathbb{Z} / 2\langle\eta q\rangle .} \\
& {\left[M_{2^{t}}^{n+1}, S^{n}\right]=\left\{\begin{array}{ll}
\mathbb{Z} / 4\left\langle\eta^{1}\right\rangle, & (n \geqslant 3) \\
\mathbb{Z} / 2\left\langle\eta^{t}\right\rangle \oplus \mathbb{Z} / 2\langle\eta \eta q\rangle, & t>1 .
\end{array} \quad(n \geqslant 3)\right.} \\
& {\left[S^{n+2}, M_{2^{t}}^{n}\right]=\left\{\begin{array}{ll}
\mathbb{Z} / 4\left\langle\xi_{1}\right\rangle, & t=1 ; \\
\mathbb{Z} / 2\left\langle\xi_{t}\right\rangle \oplus \mathbb{Z} / 2\langle i \eta \eta\rangle, & t>1 .
\end{array} \quad(n \geqslant 4)\right.}
\end{aligned}
$$

Here we choose a generator $\xi_{1}$ and set $\xi_{t}=B(\chi) \xi_{1}$. The generator $\eta^{1}$ is the dual map of $\xi_{1}$ and $\eta^{t}=\eta^{1} B(\chi)$, and $q \xi_{t}=\eta, \eta^{t} i=\eta$ for $t \geqslant 1$.

$$
\left[M_{2^{s}}^{n+1}, M_{2^{r}}^{n}\right]=\left\{\begin{array}{ll}
\mathbb{Z} / 2\left\langle\xi_{1}^{1}\right\rangle \oplus \mathbb{Z} / 2\left\langle\eta_{1}^{1}\right\rangle, & s=r=1 ; \\
\mathbb{Z} / 4\left\langle\xi_{1}^{s}\right\rangle \oplus \mathbb{Z} / 2\left\langle\eta_{1}^{s}\right\rangle, & s>1=r ; \\
\mathbb{Z} / 2\left\langle\xi_{r}^{1}\right\rangle \oplus \mathbb{Z} / 4\left\langle\eta_{r}^{1}\right\rangle, & s=1<r ; \\
\mathbb{Z} / 2\left\langle\xi_{r}^{s}\right\rangle \oplus \mathbb{Z} / 2\left\langle\eta_{r}^{s}\right\rangle \oplus \mathbb{Z} / 2\langle i \eta \eta q\rangle, & \text { otherwise, }
\end{array} \quad(n \geqslant 4)\right.
$$

where $\xi_{r}^{s}=B(\chi) \xi_{1} \in\left[M_{2^{s}}^{n+1}, M_{2^{r}}^{n}\right]_{0}^{\eta}, \eta_{r}^{s}=i \eta^{1} B(\chi) \in\left[M_{2^{s}}^{n+1}, M_{2^{r}}^{n}\right]_{\eta}^{0}$. Note that

$$
2 \xi_{1}^{s}=i \eta \eta q(s>1) ; \quad 2 \eta_{r}^{1}=i \eta \eta q(r>1) .
$$

Let $\lambda_{11}:=\xi_{r}^{s}+\eta_{r}^{s}$, then $\left[M_{2^{s}}^{n+1}, M_{2^{r}}^{n}\right]_{\eta}^{\eta}=\left\{\lambda_{11}, \lambda_{11}+i \eta \eta q\right\}$.

The group $\left[S^{n+3}, M_{2^{r}}^{n}\right]$ is given by the following lemma.

Lemma 3.1. Let $n \geqslant 5$. Then

$$
\left[S^{n+3}, M_{2}^{n}\right]=\mathbb{Z} / 2\langle i \varrho\rangle \oplus \mathbb{Z} / 2\left\langle\rho_{1}\right\rangle ;\left[S^{n+3}, M_{2^{r}}^{n}\right]=\mathbb{Z} / 4\langle i \varrho\rangle \oplus \mathbb{Z} / 2\left\langle\rho_{r}\right\rangle(r>1),
$$

where $\rho_{r}$ is some element of the group $\left[S^{n+3}, M_{2^{r}}^{n}\right]$ such that $q \rho_{r}=\eta \eta$ for $r \geqslant 1$.

Proof. The group $\left[S^{n+3}, M_{2}^{n}\right]$ is obtained from Lemma 5.2 and Theorem 5.11 of $[\mathbf{1 6}]$. For $r>1$ there are two exact sequences

$$
\begin{aligned}
& {\left[S^{k}, S^{n}\right] \stackrel{\left(2^{r}\right)^{*}}{\longrightarrow}\left[S^{k}, S^{n}\right] \rightarrow\left[S^{k}, M_{2^{r}}^{n}\right] \stackrel{q^{*}}{\longrightarrow}\left[S^{k}, S^{n+1}\right] \stackrel{\left(2^{r}\right)^{*}}{\longrightarrow}\left[S^{k}, S^{n+1}\right],} \\
& \quad k=n+2, n+3 .
\end{aligned}
$$

The following commutative diagram is induced by $\eta: S^{n+3} \rightarrow S^{n+2}$ :

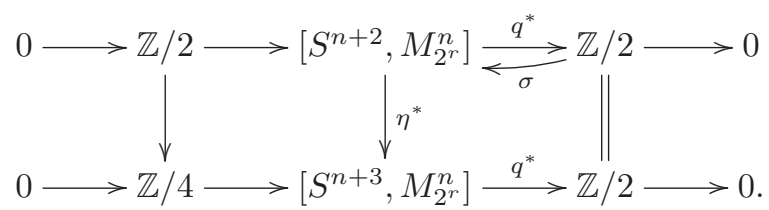

It is known that the upper exact sequence splits. If $\sigma$ is the section of $q^{*}$, then $\eta^{*} \sigma$ is the section of the lower $q^{*}$.

\subsection{Chang-complexes}

Firstly, since $C_{1}^{k, 1}$ and $\Sigma^{k-4} M_{2}^{1} \wedge M_{2}^{1}$ are both indecomposable $\mathbf{A}_{k-2}^{2}$-complexes with the same homology groups, thus $C_{1}^{k, 1} \simeq \Sigma^{k-4} M_{2}^{1} \wedge M_{2}^{1}(k \geqslant 5)$. 


\section{Cofibre sequences for Chang-complexes}

For $C \in\left\{C_{r}^{k}, C^{k, s}, C_{r}^{k, s} \mid k \geqslant 5, r, s \in \mathbb{Z}^{+}\right\}$, it can be written as mapping cones of different maps, that is, there are different cofibre sequences for $C$.

- The cofibre sequence for $C_{\eta}^{k}$

Cof : $S^{k-1} \stackrel{\eta}{\longrightarrow} S^{k-2} \stackrel{i_{\eta}}{\longrightarrow} C_{\eta}^{k} \stackrel{q_{\eta}}{\longrightarrow} S^{k} \rightarrow S^{k-1}$;

- The cofibre sequences for $C_{r}^{k}$

Cof1 : $S^{k-2} \vee S^{k-1} \stackrel{\left(2^{r}, \eta\right)}{\longrightarrow} S^{k-2} \stackrel{i_{S}}{\longrightarrow} C_{r}^{k} \stackrel{q_{S}}{\longrightarrow} S^{k-1} \vee S^{k} \rightarrow S^{k-1}$;

Cof2 : $S^{k-1} \stackrel{i \eta}{\longrightarrow} M_{2^{r}}^{k-2} \stackrel{i_{M}}{\longrightarrow} C_{r}^{k} \stackrel{q_{M}}{\longrightarrow} S^{k} \rightarrow M_{2^{r}}^{k-1}$;

Cof3 : $S^{k-2} \stackrel{i_{\eta} 2^{r}}{\longrightarrow} C_{\eta}^{k} \stackrel{i_{C}}{\longrightarrow} C_{r}^{k} \stackrel{q_{C}}{\longrightarrow} S^{k-1} \rightarrow C_{\eta}^{k+1}$;

- The cofibre sequences for $C^{k, s}$

Cof1 : $S^{k-1} \stackrel{\left(\begin{array}{c}\eta \\ 2^{s}\end{array}\right)}{\longrightarrow} S^{k-2} \vee S^{k-1} \stackrel{i_{S}}{\longrightarrow} C^{k, s} \stackrel{q_{S}}{\longrightarrow} S^{k} \rightarrow S^{k-1} \vee S^{k}$;

Cof2 : $M_{2^{s}}^{k-2} \stackrel{\eta q}{\longrightarrow} S^{k-2} \stackrel{i_{M}}{\longrightarrow} C^{k, s} \stackrel{q_{M}}{\longrightarrow} M_{2^{s}}^{k-1} \rightarrow S^{k-1}$;

Cof3 : $C_{\eta}^{k-1} \stackrel{2^{s} q_{\eta}}{\longrightarrow} S^{k-1} \stackrel{i_{C}}{\longrightarrow} C^{k, s} \stackrel{q_{C}}{\longrightarrow} C_{\eta}^{k} \rightarrow S^{k}$;

- The cofibre sequences for $C_{r}^{k, s}$

Cof1 : $S^{k-2} \vee S^{k-1} \stackrel{\left(\begin{array}{l}2^{r}, \eta \\ 0,2^{s}\end{array}\right)}{\longrightarrow} S^{k-2} \vee S^{k-1} \stackrel{i_{S}}{\longrightarrow} C_{r}^{k, s} \stackrel{q_{S}}{\longrightarrow} S^{k-1} \vee S^{k} \rightarrow S^{k-1} \vee S^{k}$;

Cof2 : $M_{2^{s}}^{k-2} \stackrel{i \eta q}{\longrightarrow} M_{2^{r}}^{k-2} \stackrel{i_{M}}{\longrightarrow} C_{r}^{k, s} \stackrel{q_{M}}{\longrightarrow} M_{2^{s}}^{k-1} \rightarrow M_{2^{r}}^{k-1}$;

Cof3 : $S^{k-2} \vee M_{2^{s}}^{k-2} \stackrel{\left(2^{r}, \eta q\right)}{\longrightarrow} S^{k-2} \stackrel{i_{\bar{M}}}{\longrightarrow} C_{r}^{k, s} \stackrel{q_{\bar{M}}}{\longrightarrow} S^{k-1} \vee M_{2^{s}}^{k-1} \rightarrow S^{k-1}$;

Cof4 : $S^{k-1} \stackrel{\left(\begin{array}{l}i \eta \\ 2^{s}\end{array}\right)}{\longrightarrow} M_{2^{r}}^{k-2} \vee S^{k-1} \stackrel{i_{\underline{M}}}{\longrightarrow} C_{r}^{k, s} \stackrel{q_{\underline{M}}}{\longrightarrow} S^{k} \rightarrow M_{2^{r}}^{k-1} \vee S^{k}$;

Cof5 : $C_{r}^{k-1} \stackrel{2^{s} p_{1} q_{S}}{\longrightarrow} S^{k-1} \stackrel{i_{\underline{C}}}{\longrightarrow} C_{r}^{k, s} \stackrel{q_{\underline{C}}}{\longrightarrow} C_{r}^{k} \rightarrow S^{k}$, where $2^{s} p_{1} q_{S}$ is the composition of $C_{r}^{k-1} \stackrel{q_{S}}{\longrightarrow} S^{k-1} \vee S^{k-2} \stackrel{p_{1}}{\longrightarrow} S^{k-1} \stackrel{2^{s}}{\longrightarrow} S^{k-1}$;

Cof6 : $S^{k-2} \stackrel{i_{S} j_{1} 2^{r}}{\longrightarrow} C^{k, s} \stackrel{i_{\bar{C}}}{\longrightarrow} C_{r}^{k, s} \stackrel{q_{\bar{C}}}{\longrightarrow} S^{k-1} \rightarrow C^{(k+1), s}$, where $i_{S} j_{1} 2^{r}$ is the composition of $S^{k-2} \stackrel{2^{r}}{\longrightarrow} S^{k-2} \stackrel{j_{1}}{\longrightarrow} S^{k-2} \vee S^{k-1} \stackrel{i_{S}}{\longrightarrow} C^{k, s}$.

\section{Homologies and cohomologies}

$$
\begin{aligned}
& H_{*} C^{k, s}= \begin{cases}\mathbb{Z}, & *=k-2 \\
\mathbb{Z} / 2^{s}, & *=k-1 \\
0, & \text { otherwise }\end{cases} \\
& H_{*} C_{r}^{k}= \begin{cases}\mathbb{Z} / 2^{r}, & *=k-2 \\
\mathbb{Z}, & *=k \\
0, & \text { otherwise; }\end{cases} \\
& H_{*} C_{r}^{k, s}= \begin{cases}\mathbb{Z} / 2^{r}, & *=k-2 \\
\mathbb{Z} / 2^{s}, & *=k-1 \\
0, & \text { otherwise }\end{cases} \\
& H_{*} C_{\eta}^{k}= \begin{cases}\mathbb{Z}, & *=k-2 \\
\mathbb{Z}, & *=k \\
0, & \text { otherwise }\end{cases} \\
& H^{*}\left(C_{r}^{k} ; \mathbb{Z} / 2\right)=H^{*}\left(C^{k, s} ; \mathbb{Z} / 2\right)= \begin{cases}\mathbb{Z} / 2, & *=k-2, k-1, k ; \\
0, & \text { otherwise }\end{cases} \\
& H^{*}\left(C_{r}^{k, s} ; \mathbb{Z} / 2\right)=\left\{\begin{array}{ll}
\mathbb{Z} / 2, & *=k-2, k ; \\
\mathbb{Z} / 2 \oplus \mathbb{Z} / 2, & *=k-1 ; \\
0, & \text { otherwise }
\end{array} \quad H^{*}\left(C_{\eta}^{k} ; \mathbb{Z} / 2\right)= \begin{cases}\mathbb{Z} / 2, & *=k-2 ; \\
\mathbb{Z} / 2, & *=k ; \\
0, & \text { otherwise }\end{cases} \right.
\end{aligned}
$$


- $S q^{2}: H^{k-2}(C ; \mathbb{Z} / 2) \rightarrow H^{k}(C ; \mathbb{Z} / 2)$ is isomorphic for $C=C^{k, s}, C_{r}^{k}, C_{r}^{k, s}, C_{\eta}^{k}$;

- $S q^{1}=0: H^{k-2}\left(C^{k, s} ; \mathbb{Z} / 2\right) \rightarrow H^{k-1}\left(C^{k, s} ; \mathbb{Z} / 2\right)$;

- $S q^{1}: H^{k-1}\left(C^{k, s} ; \mathbb{Z} / 2\right) \rightarrow H^{k}\left(C^{k, s} ; \mathbb{Z} / 2\right)$ is $\begin{cases}0, & s>1 ; \\ \text { isomorphic, } & s=1 ;\end{cases}$

- $S q^{1}: H^{k-2}\left(C_{r}^{k} ; \mathbb{Z} / 2\right) \rightarrow H^{k-1}\left(C_{r}^{k} ; \mathbb{Z} / 2\right)$ is $\begin{cases}0, & r>1 \\ \text { isomorphic, } & r=1\end{cases}$

- $S q^{1}=0: H^{k-1}\left(C_{r}^{k} ; \mathbb{Z} / 2\right) \rightarrow H^{k}\left(C_{r}^{k} ; \mathbb{Z} / 2\right)$;

- $S q^{1}$ on $H^{*}\left(C_{r}^{k, s} ; \mathbb{Z} / 2\right)$ is given by the following lemma.

Lemma 3.2. Let $v_{k-2}, v_{k}$ be generators of $H^{k-2}\left(C_{r}^{k, s} ; \mathbb{Z} / 2\right)$ and $H^{k}\left(C_{r}^{k, s} ; \mathbb{Z} / 2\right)$, respectively. Then there are generators $v_{k-1}, \bar{v}_{k-1}$ of $H^{k-1}\left(C_{r}^{k, s} ; \mathbb{Z} / 2\right)$ such that

$$
S q^{1} v_{k-2}=\left\{\begin{array}{ll}
v_{k-1}, & r=1 ; \\
0, & r>1 ;
\end{array} \quad S q^{1} \bar{v}_{k-1}=\left\{\begin{array}{ll}
v_{k}, & s=1 ; \\
0, & s>1 ;
\end{array} \quad S q^{1} v_{k-1}=0 .\right.\right.
$$

Proof. To simplify the notation, take $k=5$. Using Cof2 and Cof3 of $C_{r}^{5, s}$, we have a split exact sequence

$$
0 \rightarrow H^{4}\left(M_{2^{s}}^{4} ; \mathbb{Z} / 2\right) \stackrel{q_{M}^{*}}{\longrightarrow} H^{4}\left(C_{r}^{5, s} ; \mathbb{Z} / 2\right) \stackrel{i_{M}^{*}}{\longrightarrow} H^{4}\left(M_{2^{r}}^{3} ; \mathbb{Z} / 2\right) \rightarrow 0
$$

and an isomorphism

$$
H^{4}\left(S^{4} ; \mathbb{Z} / 2\right) \oplus H^{4}\left(M_{2^{s}}^{4} ; \mathbb{Z} / 2\right) \stackrel{\left(p_{1}^{*}, p_{2}^{*}\right) \cong}{\longrightarrow} H^{4}\left(S^{4} \vee M_{2^{s}}^{4} ; \mathbb{Z} / 2\right) \stackrel{q_{M}^{*} \cong}{\longrightarrow} H^{4}\left(C_{r}^{5, s} ; \mathbb{Z} / 2\right),
$$

where $p_{l}$ is the canonical projection for $l=1,2$. Let $u_{S}^{4}, u_{M}^{4}$ be the generators of $H^{4}\left(S^{4} ; \mathbb{Z} / 2\right)$ and $H^{4}\left(M_{2^{s}}^{4} ; \mathbb{Z} / 2\right)$, respectively. Note that $p_{2} q_{\bar{M}}=q_{M}$.

Take $\bar{v}_{4}:=q_{\bar{M}}^{*} p_{2}^{*}\left(u_{M}^{4}\right)=q_{M}^{*}\left(u_{M}^{4}\right), \quad v_{4}:= \begin{cases}S q^{1} v_{3}, & r=1, \\ q_{M}^{*} p_{1}^{*}\left(u_{S}^{4}\right), & r>1,\end{cases}$

then we can easily get this lemma.

\section{Homotopy groups and cohomotopy groups}

For $k \geqslant 5$

- $\pi_{k-1} C_{r}^{k}=0$;

- $\pi_{k} C_{r}^{k}=\mathbb{Z} / 2\left\langle\left(j_{1} \eta\right)_{S}^{-}\right\rangle \oplus \mathbb{Z}\left\langle\left(2 j_{2}\right)_{S}^{-}\right\rangle$, where $\left(j_{1} \eta\right)_{S}^{-}=q_{S *}^{-1}\left(j_{1} \eta\right)$ and $\left(2 j_{2}\right)_{S}^{-}=$ $q_{S *}^{-1}\left(2 j_{2}\right)$

- $\pi_{k-1} C^{k, s}=\mathbb{Z} / 2^{s+1}\left\langle i_{S} j_{2}\right\rangle$, with $i_{S} j_{1} \eta=2^{s} i_{S} j_{2}$;

- $\pi_{k} C^{k, s}=\mathbb{Z} / 2\left\langle i_{S} j_{2} \eta\right\rangle$;

- $\pi_{k-1} C_{r}^{k, s}=\mathbb{Z} / 2^{s+1}\left\langle i_{\underline{M}} j_{2}\right\rangle$, with $i_{\underline{M}} j_{1} i \eta=2^{s} i_{\underline{M}} j_{2}$ or $\pi_{k-1} C_{r}^{k, s}=\mathbb{Z} / 2^{s+1}\left\langle i_{S} j_{2}\right\rangle$, with $i_{S} j_{1} i \eta=2^{s} i_{S} \bar{j}_{2}$

- $\pi_{k} C_{r}^{k, s}=\mathbb{Z} / 2\left\langle i_{\underline{M}} j_{1} \xi_{r}\right\rangle \oplus \mathbb{Z} / 2\left\langle i_{\underline{M}} j_{2} \eta\right\rangle$;

- $\left[C_{r}^{k}, S^{k-2}\right]=\mathbb{Z} / 2\left\langle\eta p_{1} q_{S}\right\rangle$;

- $\left[C_{r}^{k}, S^{k-1}\right]=\mathbb{Z} / 2^{r+1}\left\langle p_{1} q_{S}\right\rangle$, with $\eta p_{2} q_{S}=2^{r} p_{1} q_{S}$;

- $\left[C^{k, s}, S^{k-2}\right]=\mathbb{Z}\left\langle\left(2 p_{1}\right)_{S}^{-}\right\rangle \oplus \mathbb{Z} / 2\left\langle\left(\eta p_{2}\right)_{S}^{-}\right\rangle$, where $\left(2 p_{1}\right)_{S}^{-}=\left(i_{S}^{*}\right)^{-1}\left(2 p_{1}\right)$ and $\left(\eta p_{2}\right)_{S}^{-}=\left(i_{S}^{*}\right)^{-1}\left(\eta p_{2}\right)$

- $\left[C^{k, s}, S^{k-1}\right]=0$; 
- $\left[C_{r}^{k, s}, S^{k-2}\right]=\mathbb{Z} / 2\left\langle\left(\eta q p_{1}\right)_{M}^{-}\right\rangle \oplus \mathbb{Z} / 2\left\langle\left(\eta p_{2}\right)_{M}^{-}\right\rangle$, where $\left(\eta q p_{1}\right)_{M}^{-}=\left(i_{M}^{*}\right)^{-1}\left(\eta q p_{1}\right)$ and $\left(\eta p_{2}\right)_{M}^{-}=\left(i_{M}^{*}\right)^{-1}\left(\eta p_{2}\right)$;

- $\left[C_{r}^{k, s}, S^{k-1}\right]=\mathbb{Z} / 2^{r+1}\left\langle p_{1} q_{S}\right\rangle$, with $\eta p_{2} q_{S}=2^{r} p_{1} q_{S}$ or $\left[C_{r}^{k, s}, S^{k-1}\right]=\mathbb{Z} / 2^{r+1}\left\langle p_{1} q_{\bar{M}}\right\rangle$, with $\eta q p_{2} q_{\bar{M}}=2^{r} p_{1} q_{\bar{M}}$

- $\left[C_{r}^{k, s}, S^{k}\right]=\mathbb{Z} / 2^{s}\left\langle q p_{2} q_{\underline{M}}\right\rangle=\mathbb{Z} / 2^{s}\left\langle p_{1} q_{S}\right\rangle$,

where $X_{u} \stackrel{j_{u}}{\longrightarrow} X_{1} \vee X_{2}$ is the canonical inclusion and $X_{1} \vee X_{2} \stackrel{p_{u}}{\longrightarrow} X_{u}$ is the canonical projection for $u=1,2$.

Remark 3.3. $[X, Y]$ for $X, Y$ being indecomposable homotopy types of $\mathbf{A}_{n}^{2}$ are given by Part IV of [1]. We will use these results directly in the rest of this paper.

\section{Determination of the decomposability except that of $C_{r}^{5, s} \wedge C_{r^{\prime}}^{5, s^{\prime}}$}

\subsection{Some indecomposable cases}

Theorem 4.1. $M_{2^{u}}^{3} \wedge C_{\eta}^{5}, C_{r}^{5} \wedge C^{5, s}, C_{r}^{5} \wedge C_{r^{\prime}}^{5}, C^{5, s} \wedge C^{5, s^{\prime}}, C_{\eta}^{5} \wedge C_{r}^{5}, C_{\eta}^{5} \wedge C^{5, s}$ and $C_{\eta}^{5} \wedge C_{r}^{5, s}$ are indecomposable for any $u, r, r^{\prime}, s, s^{\prime} \in \mathbb{Z}^{+}$.

Proof. The proofs for all cases in Theorem 4.1 are similar. We give a proof only for the case $C_{r}^{5} \wedge C_{r^{\prime}}^{5}$.

Let $u_{k}, u_{k}^{\prime}$ be generators of $H^{k}\left(C_{r}^{5} ; \mathbb{Z} / 2\right)$ and $H^{k}\left(C_{r^{\prime}}^{5} ; \mathbb{Z} / 2\right)$, respectively, for $k=$ $3,4,5$.

$$
H^{*}\left(C_{r}^{5} \wedge C_{r^{\prime}}^{5} ; \mathbb{Z} / 2\right)= \begin{cases}\mathbb{Z} / 2\left\{u_{3} \otimes u_{3}^{\prime}\right\}, & *=6, \\ \mathbb{Z} / 2\left\{u_{3} \otimes u_{4}^{\prime}, u_{4} \otimes u_{3}^{\prime}\right\}, & *=7, \\ \mathbb{Z} / 2\left\{u_{3} \otimes u_{5}^{\prime}, u_{4} \otimes u_{4}^{\prime}, u_{5} \otimes u_{3}^{\prime}\right\}, & *=8, \\ \mathbb{Z} / 2\left\{u_{4} \otimes u_{5}^{\prime}, u_{5} \otimes u_{4}^{\prime}\right\}, & *=9, \\ \mathbb{Z} / 2\left\{u_{5} \otimes u_{5}^{\prime}\right\}, & *=10, \\ 0, & \text { otherwise. }\end{cases}
$$

By the Steenrod operation action on Chang-complexes given in Section 3.2, we get

(i) $S q^{4}\left(u_{3} \otimes u_{3}^{\prime}\right)=u_{5} \otimes u_{5}^{\prime}$;

(ii) $S q^{2}\left(u_{3} \otimes u_{5}^{\prime}\right)=S q^{2}\left(u_{5} \otimes u_{3}^{\prime}\right)=u_{5} \otimes u_{5}^{\prime}$;

(iii) $S q^{2}\left(u_{3} \otimes u_{3}^{\prime}\right)= \begin{cases}u_{3} \otimes u_{5}^{\prime}+u_{4} \otimes u_{4}^{\prime}+u_{5} \otimes u_{3}^{\prime}, & r=r^{\prime}=1, \\ u_{3} \otimes u_{5}^{\prime}+u_{5} \otimes u_{3}^{\prime}, & \text { otherwise, }\end{cases}$

(iv) $S q^{2}\left(u_{4} \otimes u_{4}^{\prime}\right)=0$;

(v) $S q^{2}\left(u_{3} \otimes u_{4}^{\prime}\right)=u_{5} \otimes u_{4}^{\prime}, S q^{2}\left(u_{4} \otimes u_{3}^{\prime}\right)=u_{4} \otimes u_{5}^{\prime}$.

Suppose that $C_{r}^{5} \wedge C_{r^{\prime}}^{5}=X \vee Y$ and $H_{6} X \neq 0, C_{r}^{5} \wedge C_{r^{\prime}}^{5}$ satisfies the conditions in Lemma 2.6,

$$
H_{t} X \cong H_{t}\left(C_{r}^{5} \wedge C_{r^{\prime}}^{5}\right), \quad t=6,9,10 ; \quad \operatorname{dim} H_{7} X+\operatorname{dim} H_{8} X \geqslant 2 .
$$

If $Y$ is not contractible, then it follows from the homology groups of $C_{r}^{5} \wedge C_{r^{\prime}}^{5}$ that $Y \simeq M_{2^{x}}^{7}$ or $Y \simeq M_{2^{x}}^{8}$ for some positive integer $x$. This contradicts the isomorphism $S q^{2}: H^{7}\left(C_{r}^{5} \wedge C_{r^{\prime}}^{5} ; \mathbb{Z} / 2\right) \rightarrow H^{9}\left(C_{r}^{5} \wedge C_{r^{\prime}}^{5} ; \mathbb{Z} / 2\right)$. 
In the rest of this subsection we will give a cell structure of spaces $M_{2^{r}}^{n} \wedge C_{\eta}^{n+2}$ $(n \geqslant 3)$ and $C_{\eta}^{5} \wedge C_{r}^{5, s}$ which will be used later.

Lemma 4.2. $M_{2^{r}}^{n} \wedge C_{\eta}^{n+2} \simeq S^{2 n} \cup_{h^{r}} \mathbf{C} C^{(2 n+2), r} \simeq C_{r}^{2 n+2} \cup_{h_{r}} \mathbf{C} S^{2 n+2}(n \geqslant 3)$, where $h^{r}$ is determined by $h^{r} i_{S}=\left(2^{r}, \eta\right)$, i.e.,

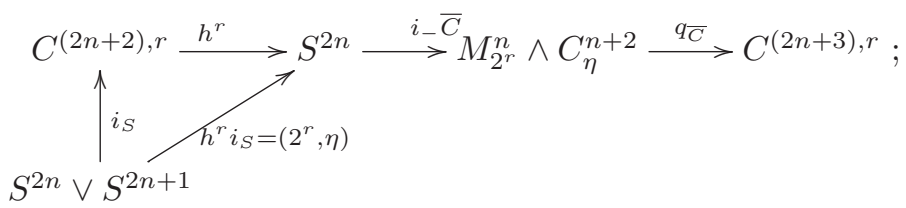

$h_{r}$ is determined by $q_{S} h_{r}=\left(\begin{array}{c}\eta \\ 2^{r}\end{array}\right)$, i.e.,

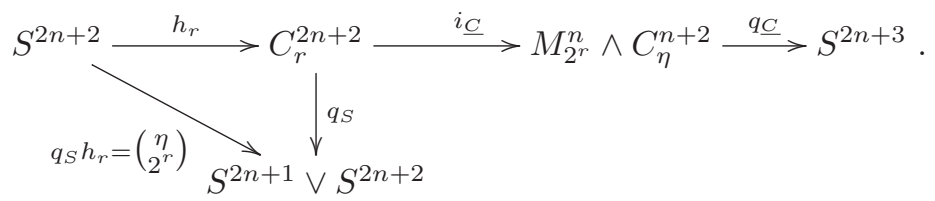

The top rows are cofibre sequences in each commutative diagram.

Moreover,

$$
\begin{aligned}
& \pi_{2 n+1}\left(M_{2^{r}}^{n} \wedge C_{\eta}^{n+2}\right)=\left[M_{2^{r}}^{n} \wedge C_{\eta}^{n+2}, S^{2 n+2}\right]=0 ; \\
& \mathbb{Z} / 2^{r} \cong \frac{\pi_{2 n+2} C_{r}^{2 n+2}}{\left\langle\left(j_{1} \eta\right)_{S}^{-}, 2^{r-1}\left(2 j_{2}\right)_{S}^{-}\right\rangle} \stackrel{\cong}{\left(i_{\underline{C}}\right)_{*}} \pi_{2 n+2}\left(M_{2^{r}}^{n} \wedge C_{\eta}^{n+2}\right) ; \\
& \mathbb{Z} / 2^{r} \cong \frac{\left[C^{(2 n+3), r}, S^{2 n+1}\right]}{\left\langle\left(\eta p_{2}\right)_{S}^{-}, 2^{r-1}\left(2 p_{1}\right)_{S}^{-}\right\rangle} \underset{\left(q_{\underline{C}}\right)^{*}}{\cong}\left[M_{2^{r}}^{n} \wedge C_{\eta}^{n+2}, S^{2 n+1}\right] ; \\
& \frac{\pi_{2 n+3} S^{2 n}}{\left\langle 2^{r} \varrho_{2 n}, \eta^{(3)}\right\rangle} \underset{\left(i_{\bar{C}}\right)_{*}}{\cong} \pi_{2 n+3}\left(M_{2^{r}}^{n} \wedge C_{\eta}^{n+2}\right) ; \quad \frac{\left[S^{2 n+3}, S^{2 n}\right]}{\left\langle 2^{r} \varrho_{2 n}, \eta^{(3)}\right\rangle} \underset{\left(q_{\bar{C}}\right)^{*}}{\cong}\left[M_{2^{r}}^{n} \wedge C_{\eta}^{n+2}, S^{2 n}\right] ;
\end{aligned}
$$

Proof. From Lemma 2.4

$$
\begin{gathered}
\left(M_{2^{r}}^{n} \wedge C_{\eta}^{n+2}\right) / S^{2 n} \simeq\left(S^{2 n+1} \vee S^{2 n+2}\right) \cup{ }_{\left(\begin{array}{c}
\eta \\
-2^{r}
\end{array}\right)} \mathbf{C} S^{2 n+2} \simeq C^{(2 n+3), r} ; \\
\left(M_{2^{r}}^{n} \wedge C_{\eta}^{n+2}\right)^{(2 n+2)} \simeq S^{2 n} \cup_{\left(2^{r}, \eta\right)} \mathbf{C}\left(S^{2 n} \vee S^{2 n+1}\right) \simeq C_{r}^{2 n+2} .
\end{gathered}
$$

So there are cofibre sequences

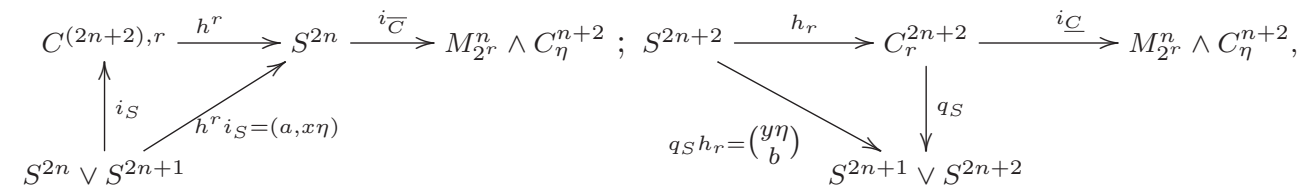

where $a, b \in \mathbb{Z}$ and $x, y \in\{0,1\}$. It follows from the monomorphisms $\left[C^{(2 n+2), r}, S^{2 n}\right]$ $\stackrel{i_{S}^{*}}{\longrightarrow}\left[S^{2 n} \vee S^{2 n+1}, S^{2 n}\right]$ and $\left[S^{2 n+2}, C_{r}^{2 n+2}\right] \stackrel{\left(q_{S}\right)_{*}}{\longrightarrow}\left[S^{2 n+2}, S^{2 n+1} \vee S^{2 n+2}\right]$ that $h^{r}$ and $h_{r}$ are determined by $h^{r} i_{S}$ and $q_{S} h_{r}$, respectively. 
By $H_{2 n}\left(M_{2^{r}}^{n} \wedge C_{\eta}^{n+2}\right)=\mathbb{Z} / 2^{r}$ and $\pi_{2 n+1}\left(M_{2^{r}}^{n} \wedge C_{\eta}^{n+2}\right) \cong \pi_{2 n+1}\left(C_{r}^{2 n+2}\right)=0$,

$$
a=2^{r}, x=1 .
$$

By $H_{2 n+2}\left(M_{2^{r}}^{n} \wedge C_{\eta}^{n+2}\right)=\mathbb{Z} / 2^{r}$ and $\left[M_{2^{r}}^{n} \wedge C_{\eta}^{n+2}, S^{2 n+2}\right] \cong\left[C^{(2 n+3), r}, S^{2 n+2}\right]=0$

$$
b=2^{r}, y=1 .
$$

Thus we prove the first part of this lemma. Now $\pi_{*}\left(M_{2^{r}}^{n} \wedge C_{\eta}^{n+2}\right)(*=2 n+1,2 n+$ $2,2 n+3)$ and $\left[M_{2^{r}}^{n} \wedge C_{\eta}^{n+2}, S^{m}\right](m=2 n, 2 n+1,2 n+2)$ are easily obtained.

Lemma 4.3. $C_{\eta}^{5} \wedge C_{r}^{5, s}$ is homotopy equivalent to the mapping cone of map

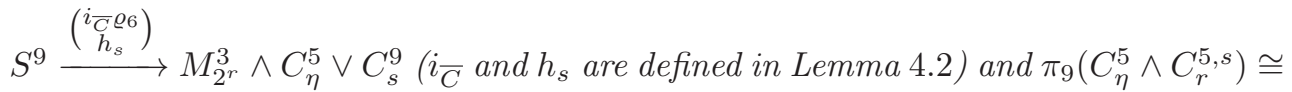
$\begin{cases}\mathbb{Z} / 2^{s+1} \oplus \mathbb{Z} / 2, & r>1, \\ \mathbb{Z} / 2^{s} \oplus \mathbb{Z} / 2, & r=1 .\end{cases}$

Proof. Apply Lemma 2.4 to the cofibre sequences

$$
\begin{gathered}
S^{4} \stackrel{\eta}{\rightarrow} S_{a}^{3} \rightarrow C_{\eta}^{5} ; \quad S^{3} \vee M_{2^{s}}^{3} \stackrel{f=\left(2^{r}, \eta q\right)}{\longrightarrow} S_{b}^{4} \rightarrow C_{r}^{5, s} ; \\
\left(C_{\eta}^{5} \wedge C_{r}^{5, s}\right) / S^{6} \simeq\left(\Sigma\left(S^{4} \wedge S_{b}^{3}\right) \vee \Sigma\left(S_{a}^{3} \wedge\left(S^{3} \vee M_{2^{s}}^{3}\right)\right)\right) \cup_{\mathcal{A}} \mathbf{C} \Sigma S^{4} \wedge\left(S^{3} \vee M_{2^{s}}^{3}\right),
\end{gathered}
$$

where $\mathcal{A}=\left(\begin{array}{c}\Sigma 1 \wedge f \\ -\Sigma \eta \wedge 1\end{array}\right)$, i.e.,

$$
\left(C_{\eta}^{5} \wedge C_{r}^{5, s}\right) / S^{6} \simeq\left(S^{8} \vee S^{7} \vee M_{2^{s}}^{7}\right) \cup_{\mathcal{A}} \mathbf{C}\left(S^{8} \vee M_{2^{s}}^{8}\right)
$$

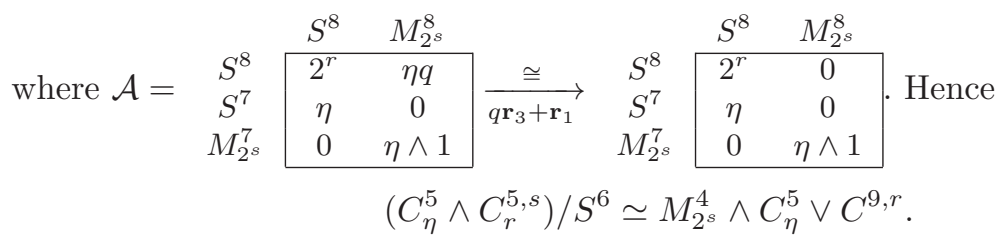

Apply Lemma 2.4 to the cofibre sequences

$$
\begin{gathered}
S_{1}^{4} \stackrel{\eta}{\rightarrow} S^{3} \rightarrow C_{\eta}^{5} ; \quad S_{2}^{4} \stackrel{g=\left(\begin{array}{l}
i \eta \\
2^{s}
\end{array}\right)}{\longrightarrow} M_{2^{r}}^{3} \vee S_{a}^{4} \rightarrow C_{r}^{5, s} ; \\
\left(C_{\eta}^{5} \wedge C_{r}^{5, s}\right)^{(9)} \simeq\left(M_{2^{r}}^{6} \vee S^{7}\right) \cup_{\mathcal{B}} \mathbf{C}\left(M_{2^{r}}^{7} \vee S^{8} \vee S^{7}\right),
\end{gathered}
$$

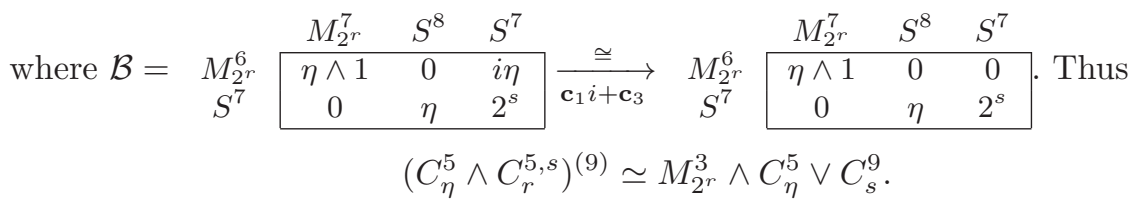

There is a cofibre sequence

$$
S^{9} \stackrel{\left(\begin{array}{c}
\alpha^{\prime} \\
\beta^{\prime}
\end{array}\right)}{\longrightarrow} M_{2^{r}}^{3} \wedge C_{\eta}^{5} \vee C_{s}^{9} \rightarrow C_{\eta}^{5} \wedge C_{r}^{5, s} \rightarrow S^{10} \stackrel{\left(\begin{array}{c}
\Sigma \alpha^{\prime} \\
\Sigma \beta^{\prime}
\end{array}\right)}{\longrightarrow} M_{2^{r}}^{4} \wedge C_{\eta}^{5} \vee C_{s}^{10},
$$

where $\alpha^{\prime}=i_{\bar{C}}\left(t^{\prime} \varrho_{6}\right), \quad t^{\prime}=1 \quad$ for $\quad r=1 \quad$ and $\quad t^{\prime} \in\{1,2\} \quad$ for $\quad r>1$ since $\pi_{9}\left(M_{2^{r}}^{3} \wedge C_{\eta}^{5}\right)=\left\{\begin{array}{ll}\mathbb{Z} / 2\left\langle i_{\bar{C}} \varrho_{6}\right\rangle, & r=1 \\ \mathbb{Z} / 4\left\langle i_{\bar{C}} \varrho_{6}\right\rangle, & r \geqslant 2\end{array}\right.$ and $C_{\eta}^{5} \wedge C_{r}^{5, s}$ is indecomposable. $\beta^{\prime}$ is 
determined by $q_{S} \beta^{\prime}=\left(\begin{array}{c}y^{\prime} \eta \\ b^{\prime}\end{array}\right) \quad$ for $\quad$ some $\quad y^{\prime} \in\{0,1\} \quad$ and $\quad b^{\prime} \in \mathbb{Z} \quad$ since $\left(q_{S}\right)_{*}: \pi_{9} C_{s}^{9} \rightarrow \pi_{9}\left(S^{8} \vee S^{9}\right)$ is injective.
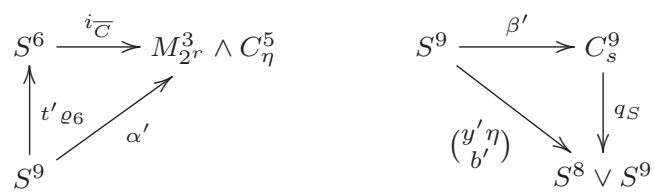

By $H_{9}\left(C_{\eta}^{5} \wedge C_{r}^{5, s}\right)=\mathbb{Z} / 2^{s}, b^{\prime}=2^{s}$. From $\left[C_{\eta}^{5} \wedge C_{r}^{5, s}, S^{9}\right] \cong\left[\left(C_{\eta}^{5} \wedge C_{r}^{5, s}\right) / S^{6}, S^{9}\right]=$ $\left[M_{2^{s}}^{4} \wedge C_{\eta}^{5} \vee C^{9, r}, S^{9}\right] \cong \mathbb{Z} / 2^{r}$ and the exact sequence

$$
0 \rightarrow \frac{\left[S^{10}, S^{9}\right]}{\left\langle y^{\prime} \eta\right\rangle} \rightarrow\left[C_{\eta}^{5} \wedge C_{r}^{5, s}, S^{9}\right] \rightarrow \mathbb{Z} / 2^{r} \rightarrow 0
$$

we have $y^{\prime}=1$. Thus $\beta^{\prime}=h_{s}$. So for $r=1$,

$$
\begin{aligned}
\pi_{9}\left(C_{\eta}^{5} \wedge C_{r}^{5, s}\right) & \cong \frac{\mathbb{Z} / 2\left\langle i_{\bar{C}} \varrho_{6}\right\rangle \oplus \mathbb{Z} / 2\left\langle\left(i_{1} \eta\right)^{-}\right\rangle \oplus \mathbb{Z}\left\langle\left(2 i_{2}\right)^{-}\right\rangle}{\left\langle\left(t^{\prime} i_{\bar{C}} \varrho_{6},\left(i_{1} \eta\right)^{-}, 2^{s-1}\left(2 i_{2}\right)^{-}\right)\right\rangle} \\
& \cong \frac{\mathbb{Z} / 2 \oplus \mathbb{Z} / 2 \oplus \mathbb{Z}}{\left\langle\left(1,1,2^{s-1}\right)\right\rangle} \cong \mathbb{Z} / 2 \oplus \mathbb{Z} / 2^{s} .
\end{aligned}
$$

Next we will determine $t^{\prime}$ for $r>1$.

By computing the exact sequence $\pi_{9}$ of cofibre sequence (1), we get

$$
\pi_{9}\left(C_{\eta}^{5} \wedge C_{r}^{5, s}\right) \cong \frac{\mathbb{Z} / 4 \oplus \mathbb{Z} / 2 \oplus \mathbb{Z}}{\left\langle\left(t^{\prime}, 1,2^{s-1}\right)\right\rangle} \cong \begin{cases}\mathbb{Z} / 4 \oplus \mathbb{Z} / 2^{s}, & t^{\prime}=2 \\ \mathbb{Z} / 2 \oplus \mathbb{Z} / 2^{s+1}, & t^{\prime}=1\end{cases}
$$

On the other hand, $\pi_{9}\left(C_{\eta}^{5} \wedge C_{r}^{5, s}\right) \cong\left[C_{\eta}^{14}, C_{r}^{13, s}\right] \cong\left[C_{\eta}^{7}, C_{r}^{6, s}\right]\left(\left[C_{\eta}^{k+1}, C_{r}^{k, s}\right]\right.$ is stable for $k \geqslant 6)$. There is an exact sequence

$$
\left[C_{\eta}^{7}, S^{5}\right] \stackrel{\left(\begin{array}{l}
i \eta \\
2^{s}
\end{array}\right)}{\longrightarrow}\left[C_{\eta}^{7}, M_{2^{r}}^{4} \vee S^{5}\right] \rightarrow\left[C_{\eta}^{7}, C_{r}^{6, s}\right] \rightarrow\left[C_{\eta}^{7}, S^{6}\right]=0
$$

By Lemma 3.1 , we know that $\mathbb{Z} / 12 \cong\left[C_{\eta}^{7}, S^{4}\right] \stackrel{i_{*}}{\rightarrow}\left[C_{\eta}^{7}, M_{2^{r}}^{4}\right] \cong \mathbb{Z} / 4$ is an epimorphism. By $\left[C_{\eta}^{7}, C_{\eta}^{6}\right] \cong \frac{\left[C_{\eta}^{7}, S^{4}\right]}{\eta_{*}\left[C_{\eta}^{7}, S^{5}\right]} \cong \frac{\left[S^{7}, C_{\eta}^{6}\right]}{\eta^{*}\left[S^{6}, C_{\eta}^{6}\right]} \cong \mathbb{Z} / 6[\mathbf{1 5}$, Proposition 2.6 (iii)], we get $\mathbb{Z}=\left[C_{\eta}^{7}, S^{5}\right] \stackrel{\eta_{*}=6}{\longrightarrow}\left[C_{\eta}^{7}, S^{4}\right]=\mathbb{Z} / 12$, which implies

$$
(i \eta)_{*}=2: \mathbb{Z}=\left[C_{\eta}^{7}, S^{5}\right] \stackrel{\eta_{*}}{\longrightarrow}\left[C_{\eta}^{7}, S^{4}\right] \stackrel{i_{*}}{\longrightarrow}\left[C_{\eta}^{7}, M_{2^{r}}^{4}\right] \cong \mathbb{Z} / 4 .
$$

Hence

$$
\pi_{9}\left(C_{\eta}^{5} \wedge C_{r}^{5, s}\right) \cong\left[C_{\eta}^{7}, C_{r}^{6, s}\right] \cong \frac{\mathbb{Z} / 4 \oplus \mathbb{Z}}{\left\langle\left(2,2^{s}\right)\right\rangle} \cong \mathbb{Z} / 2^{s+1} \oplus \mathbb{Z} / 2 .
$$

Together with $(2), t^{\prime}=1$.

4.2. $M_{2^{u}}^{3} \wedge C, C \in\left\{C_{r}^{5}, C^{5, s}, C_{r}^{5, s} \mid r, s \in \mathbb{Z}^{+}\right\}$

(1) $M_{2^{u}}^{3} \wedge C_{r}^{5}$ and $M_{2^{u}}^{3} \wedge C^{5, r}$ 
There is the following commutative diagram:

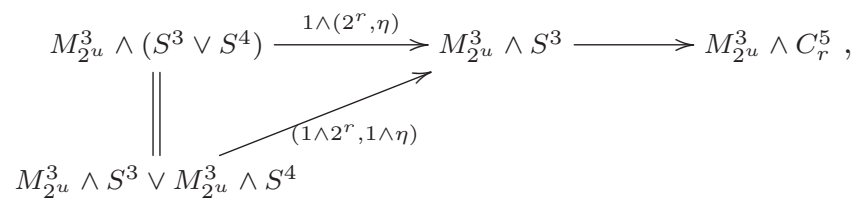

where the top row is a cofibre sequence. Hence

$$
M_{2^{u}}^{3} \wedge C_{r}^{5} \simeq M_{2^{u}}^{3} \wedge S^{3} \cup_{\left(1 \wedge 2^{r}, 1 \wedge \eta\right)} \mathbf{C}\left(M_{2^{u}}^{3} \wedge S^{3} \vee M_{2^{u}}^{3} \wedge S^{4}\right) .
$$

- If $r \geqslant u$ and $r>1$, then $1 \wedge 2^{r} \simeq 0$, hence $M_{2^{u}}^{3} \wedge C_{r}^{5} \simeq M_{2^{u}}^{7} \vee M_{2^{u}}^{3} \wedge C_{\eta}^{5}$.

- If $r=u=1,1 \wedge \eta \in\left[M_{2^{u}}^{7}, M_{2^{u}}^{6}\right]_{\eta}^{\eta}$ implies $1 \wedge \eta=\lambda_{11}+\varepsilon_{u} i \eta \eta q$ for some $\varepsilon_{u}$ $\in\{0,1\}$ (for $u=1, \varepsilon_{1}=0$, i.e., $\lambda_{11}=1 \wedge \eta$ ), then

$$
M_{2}^{3} \wedge C_{1}^{5} \simeq M_{2}^{6} \cup_{\mathcal{M}=\left(i_{6} \eta q_{6}, \lambda_{11}\right)} \mathbf{C}\left(M_{2}^{6} \vee M_{2}^{7}\right) \text {. }
$$

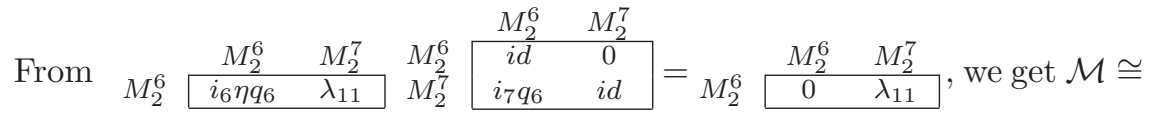
$\left(0, \lambda_{11}\right)$, thus $M_{2}^{3} \wedge C_{1}^{5} \simeq M_{2}^{7} \vee M_{2}^{3} \wedge C_{\eta}^{5}$.

- If $r<u$, apply Lemma 2.4 to the cofibre sequences

$$
S_{1}^{3} \stackrel{2^{u}}{\longrightarrow} S_{a}^{3} \rightarrow M_{2^{u}}^{3} ; \quad S_{2}^{3} \vee S^{4} \stackrel{\left(2^{r}, \eta\right)}{\longrightarrow} S_{b}^{3} \rightarrow C_{r}^{5},
$$

then we can easily get

$$
\left(M_{2^{u}}^{3} \wedge C_{r}^{5}\right) / S^{6} \simeq S^{7} \vee C_{r}^{9, u}
$$

Suppose that $M_{2^{u}}^{3} \wedge C_{r}^{5} \simeq X \vee Y$ are decomposable and $H_{6}(X) \neq 0$.

Since $S q^{2}$ on $H^{6}\left(M_{2^{u}}^{3} \wedge C_{r}^{5} ; \mathbb{Z} / 2\right)$ and $H^{7}\left(M_{2^{u}}^{3} \wedge C_{r}^{5} ; \mathbb{Z} / 2\right)$ are nontrivial, $M_{2^{u}}^{3} \wedge C_{r}^{5}$ has no direct summands $M_{2^{r}}^{6}$ and $M_{2^{u}}^{8}$. Thus $M_{2^{u}}^{3} \wedge C_{r}^{5} \simeq X \vee$ $M_{2^{r}}^{7}$. By Lemma 2.6 and (5), $\left(X / S^{6}\right) \vee M_{2^{r}}^{7} \simeq S^{7} \vee C_{r}^{9, u}$ which contradicts the uniqueness of the decomposability of $\mathbf{A}_{n}^{3}$-complexes [5]. So $M_{2^{u}}^{3} \wedge C_{r}^{5}$ is indecomposable for $r<u$.

In summary, $M_{2^{u}}^{3} \wedge C_{r}^{5}$ is homotopy equivalent to $M_{2^{u}}^{3} \wedge C_{\eta}^{5} \vee M_{2^{u}}^{7}$ for $r \geqslant u$ and indecomposable for $u>r$. Thus $M_{2^{u}}^{3} \wedge C^{5, r} \simeq D\left(M_{2^{u}}^{4} \wedge C_{r}^{5}\right)$ is homotopy equivalent to $M_{2^{u}}^{3} \wedge C_{\eta}^{5} \vee M_{2^{u}}^{7}$ for $r \geqslant u$ and indecomposable for $u>r$.

(2) $M_{2^{u}}^{3} \wedge C_{r}^{5, s}$

If $u>r$ and $u>s$ there is a cofibre sequence

$$
S^{3} \wedge C_{r}^{5, s} \stackrel{2^{u} \wedge 1}{\longrightarrow} S^{3} \wedge C_{r}^{5, s} \rightarrow M_{2^{u}}^{3} \wedge C_{r}^{5, s},
$$

$\left[C_{r}^{k, s}, C_{r}^{k, s}\right] \cong \mathbb{Z} / 2^{\max (r, s)+1} \oplus \mathbb{Z} / 2^{m_{s r}} \oplus \mathbb{Z} / 2^{m_{s r}+1}$ for $k \geqslant 5$ which implies $2^{u} \wedge$ $1=0 \in\left[S^{3} \wedge C_{r}^{5, s}, S^{3} \wedge C_{r}^{5, s}\right]$. Thus $M_{2^{u}}^{3} \wedge C_{r}^{5, s} \simeq C_{r}^{8, s} \vee C_{r}^{9, s}$.

If $u \leqslant r$ and $r>1$, by $2^{r}=0 \in\left[M_{2^{u}}^{6}, M_{2^{u}}^{6}\right]$ and the following cofibre sequence

$$
M_{2^{u}}^{3} \wedge\left(S^{3} \vee M_{2^{s}}^{3} \stackrel{1 \wedge\left(2^{r}, \eta q\right)}{\longrightarrow} M_{2^{u}}^{3} \wedge S^{3} \rightarrow M_{2^{u}}^{3} \wedge C_{r}^{5, s},\right.
$$

we get $M_{2^{u}}^{3} \wedge C_{r}^{5, s} \simeq M_{2^{u}}^{7} \vee \mathbf{C}_{1 \wedge \eta q} \simeq M_{2^{u}}^{3} \wedge C^{5, s} \vee M_{2^{u}}^{7}$. So

$$
M_{2^{u}}^{3} \wedge C_{r}^{5, s} \simeq \begin{cases}M_{2^{u}}^{3} \wedge C^{5, s} \vee M_{2^{u}}^{7}, & s<u \leqslant r, \\ M_{2^{u}}^{3} \wedge C_{\eta}^{5} \vee M_{2^{u}}^{7} \vee M_{2^{u}}^{7}, & u \leqslant r, s \text { and } r>1 .\end{cases}
$$


If $u \leqslant s$ and $s>1, M_{2^{u}}^{3} \wedge C_{r}^{5, s} \simeq D\left(M_{2^{u}}^{4} \wedge C_{s}^{5 r}\right)$, we get

$$
M_{2^{u}}^{3} \wedge C_{r}^{5, s} \simeq \begin{cases}M_{2^{u}}^{3} \wedge C_{r}^{5} \vee M_{2^{u}}^{7}, & r<u \leqslant s \\ M_{2^{u}}^{3} \wedge C_{\eta}^{5} \vee M_{2^{u}}^{7} \vee M_{2^{u}}^{7}, & u \leqslant r, s \text { and } s>1 .\end{cases}
$$

If $u=r=s=1$, then from Corollary 3.7 of [16],

$$
M_{2}^{3} \wedge C_{1}^{5,1} \simeq \Sigma M_{2}^{3} \wedge M_{2}^{1} \wedge M_{2}^{1} \simeq M_{2}^{3} \wedge C_{\eta}^{5} \vee M_{2}^{7} \vee M_{2}^{7}
$$

4.3. $C_{u}^{5} \wedge C_{r}^{5, s}$ and $C^{5, u} \wedge C_{r}^{5, s}$ for $u, r, s \in \mathbb{Z}^{+}$

We only prove the case $C_{u}^{5} \wedge C_{r}^{5, s}$ since $C^{5, u} \wedge C_{r}^{5, s} \simeq D\left(C_{u}^{5} \wedge C_{s}^{5, r}\right)$.

Let $m_{u v}$ be the minimum of non-negative integers $u$ and $v$.

The following corollary is easy to get from Lemma 2.5.

Corollary 4.4. If $C_{u}^{5} \wedge C_{r}^{5, s} \simeq X \vee Y$ is decomposable and $H_{6}(X) \neq 0$, then $X$ is indecomposable and $Y \simeq C_{l}^{9, t}$ for some $t \in\left\{m_{u s}, r\right\}, l \in\left\{m_{u r}, m_{u s}\right\}$.

Firstly, we study $\left(C_{u}^{5} \wedge C_{r}^{5, s}\right) / S^{6}$.

Applying Lemma 2.4 to the following cofibre sequences

$$
\begin{aligned}
& S_{1}^{3} \vee S^{4} \stackrel{\left(2^{u}, \eta\right)}{\longrightarrow} S_{a}^{3} \rightarrow C_{u}^{5} ; \quad S_{2}^{3} \vee M_{2^{s}}^{3} \stackrel{\left(2^{r}, \eta q\right)}{\longrightarrow} S_{b}^{3} \rightarrow C_{r}^{5, s}, \\
& \left(C_{u}^{5} \wedge C_{r}^{5, s}\right) / S^{6} \simeq\left(S^{7} \vee S^{8} \vee S^{7} \vee M_{2^{s}}^{7}\right) \cup_{\mathcal{A}} \mathbf{C}\left(S^{7} \vee S^{8} \vee M_{2^{s}}^{7} \vee M_{2^{s}}^{8}\right),
\end{aligned}
$$

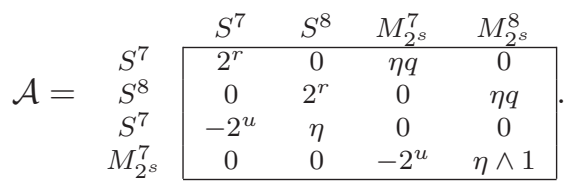

Note that $q(\eta \wedge 1)=\eta q$ for $u \geqslant s ; 2^{u}=\left\{\begin{array}{ll}0, & u>1 \\ i \eta q, & u=s=1\end{array}\right.$ in $\left[M_{2^{s}}^{7}, M_{2^{s}}^{7}\right]$.

For $s \leqslant u<r$, by transformations $i \mathbf{r}_{1}+\mathbf{r}_{4}$ if $u=1$ (otherwise, omitting this one); $q \mathbf{r}_{4}+\mathbf{r}_{2} ; 2^{r-u} \mathbf{r}_{3}+\mathbf{r}_{1}$ and $-\mathbf{r}_{3}$,

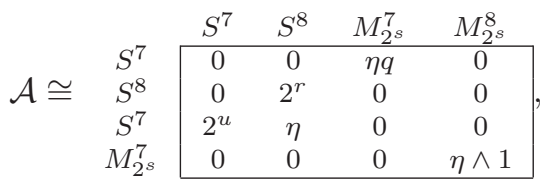

$$
\begin{aligned}
& \left(C_{u}^{5} \wedge C_{r}^{5, s}\right) / S^{6} \simeq C_{u}^{9, r} \vee C^{9, s} \vee M_{2^{s}}^{4} \wedge C_{\eta}^{5} \quad(s \leqslant u<r) .
\end{aligned}
$$

Similarly,

$$
\begin{aligned}
& \left(C_{u}^{5} \wedge C_{r}^{5, s}\right) / S^{6} \simeq C_{r}^{9, s} \vee C^{9, r} \vee M_{2^{s}}^{4} \wedge C_{\eta}^{5} \quad(s \leqslant u \text { and } r \leqslant u), \\
& \left(C_{u}^{5} \wedge C_{r}^{5, s}\right) / S^{6} \simeq C^{9, r} \vee \mathbf{C}_{\mathcal{A}^{\prime}} \quad(r \leqslant u<s), \\
& \left(C_{u}^{5} \wedge C_{r}^{5, s}\right) / S^{6} \simeq \mathbf{C}_{\mathcal{A}^{\prime \prime}} \quad(r \leqslant u<s),
\end{aligned}
$$

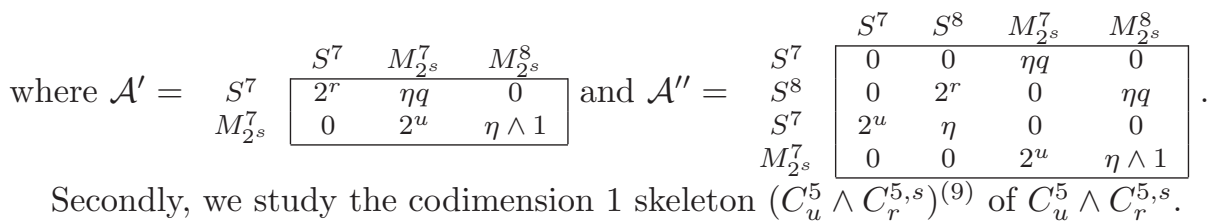


Using the cofibre sequences

$$
S_{1}^{4} \stackrel{i \eta}{\longrightarrow} M_{2^{u}}^{3} \rightarrow C_{u}^{5} ; \quad S_{2}^{4} \stackrel{g=\left(\begin{array}{l}
i \eta \\
2^{s}
\end{array}\right)}{\longrightarrow} M_{2^{r}}^{3} \vee S_{b}^{4} \rightarrow C_{r}^{5, s},
$$

we get

$$
\left(C_{u}^{5} \wedge C_{r}^{5, s}\right)^{(9)} \simeq\left(M_{2^{u}}^{3} \wedge S_{b}^{4} \vee M_{2^{u}}^{3} \wedge M_{2^{r}}^{3}\right) \cup_{\mathcal{B}} \mathbf{C}\left(S_{1}^{4} \wedge S_{b}^{4} \vee S_{1}^{4} \wedge M_{2^{r}}^{3} \vee M_{2^{u}}^{3} \wedge S_{2}^{4}\right),
$$

where

$$
\mathcal{B}=\begin{array}{cccc} 
& S_{1}^{4} \wedge S_{b}^{4} & S_{1}^{4} \wedge M_{2^{r}}^{3} & M_{2^{u}}^{3} \wedge S_{2}^{4} \\
\cline { 2 - 4 } M_{2^{u}}^{3} \wedge S_{b}^{4} & i \eta \wedge 1 & i \eta \wedge 0 & 1 \wedge 2^{s} \\
M_{2^{u}}^{3} \wedge M_{2^{r}}^{3} & i \eta \wedge 0 & i \eta \wedge 1 & 1 \wedge i \eta \\
\cline { 3 - 4 } & &
\end{array}
$$

Let

$$
\mathcal{B}_{1}=\begin{array}{cc}
S_{1}^{4} \wedge M_{2^{r}}^{3} & M_{2^{u}}^{3} \wedge S_{2}^{4} \\
\hline i \eta 2^{u} \wedge M_{2^{r}}^{3} & i \wedge i \eta \\
\hline
\end{array}
$$

For $u \geqslant r$ and $u>1$, by Lemma 6.2 .1 of [10], there is a retraction $\tau_{3}^{\prime}$ of $i \wedge 1$ yielding the following commutative diagram:

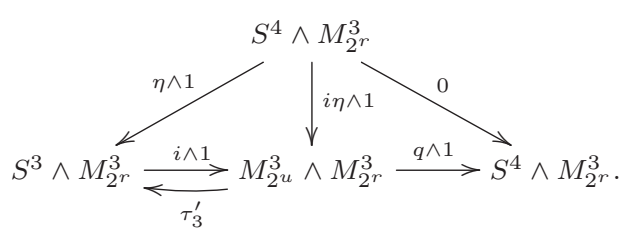

From the following commutative diagrams:
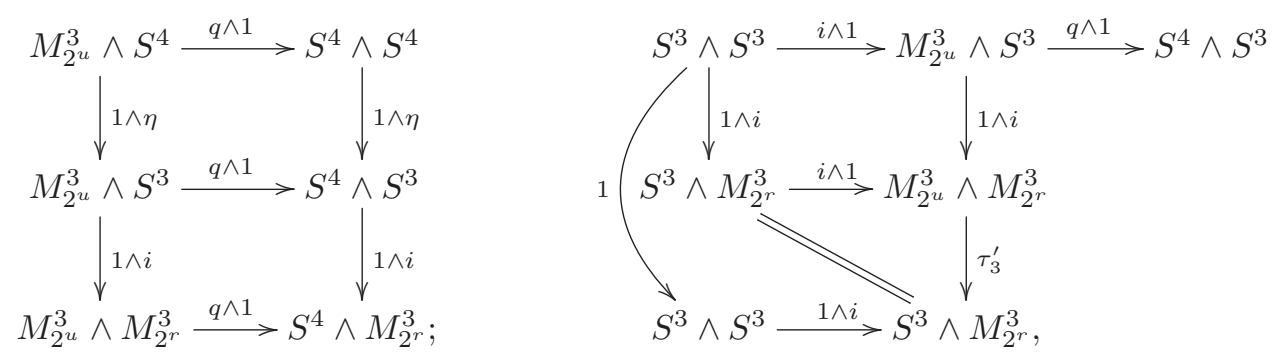

we get $(q \wedge 1)(1 \wedge i \eta)=i \eta q \in\left[M_{2^{u}}^{7}, M_{2^{r}}^{7}\right] ; \tau_{3}^{\prime}(1 \wedge i) \in\left[M_{2^{u}}^{6}, M_{2^{r}}^{6}\right]_{1}^{2^{u-r}}$.

So $\tau_{3}^{\prime}(1 \wedge i \eta) \in\left[M_{2^{u}}^{7}, M_{2^{r}}^{6}\right]_{\eta}^{\eta}$ for $u=r$ and $\tau_{3}^{\prime}(1 \wedge i \eta) \in\left[M_{2^{u}}^{7}, M_{2^{r}}^{6}\right]_{\eta}^{0}$ for $u>r$. Since $\eta \wedge 1: S^{4} \wedge M_{2^{r}}^{3} \rightarrow S^{3} \wedge M_{2^{r}}^{3}$ is also an element in $\left[M_{2^{r}}^{7}, M_{2^{r}}^{6}\right]_{\eta}^{\eta}$,

$$
\tau_{3}^{\prime}(1 \wedge i \eta)= \begin{cases}\eta \wedge 1+\kappa i \eta \eta q, & r=u>1 \\ \eta_{r}^{u}+\kappa i \eta \eta q, & u>r\end{cases}
$$

Note that, since the composition $M_{2^{u}}^{7} \stackrel{B(\chi)}{\longrightarrow} M_{2^{r}}^{7} \stackrel{\eta \wedge 1}{\longrightarrow} M_{2^{r}}^{6}(u>r)$ is an element in $\left[M_{2^{u}}^{7}, M_{2^{r}}^{6}\right]_{\eta}^{0},(\eta \wedge 1) B(\chi)=\eta_{r}^{u}+\kappa^{\prime} i \eta \eta q$, which implies that

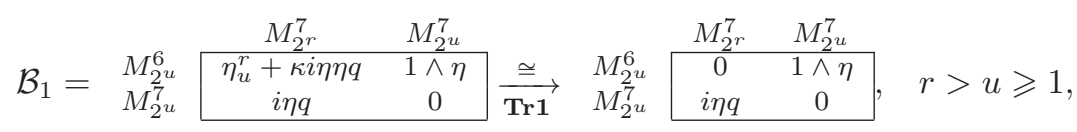


where the invertible transformation $\operatorname{Tr} 1$ is $\mathbf{c}_{2}\left(-B(\chi)-\left(\kappa+\kappa^{\prime}\right) i \eta q\right)+\mathbf{c}_{1}$. Similarly,

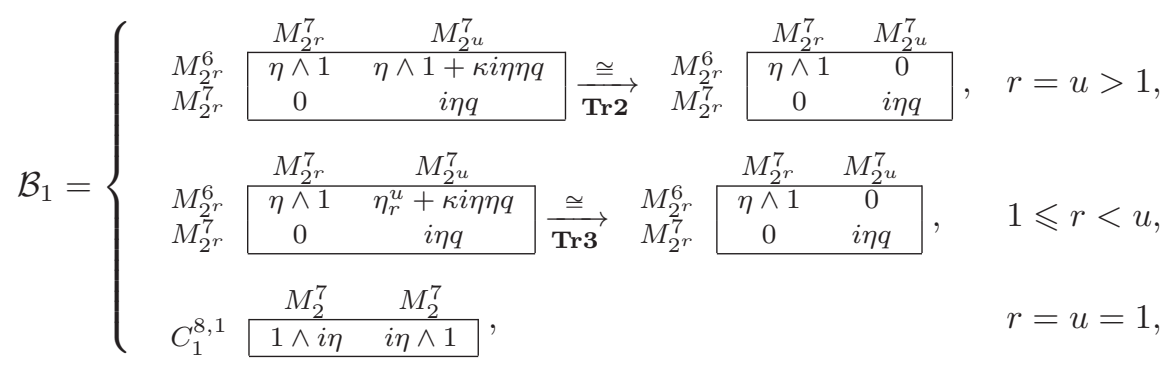

where the invertible transformations are given by

$$
\operatorname{Tr} 2: \mathbf{c}_{1}(1+\kappa i \eta q)+\mathbf{c}_{2} ; \quad \operatorname{Tr} \mathbf{3}: \mathbf{c}_{1}\left(-B(\chi)-\left(\kappa+\kappa^{\prime}\right) i \eta q\right)+\mathbf{c}_{2} .
$$

- For $s \geqslant u$, since $2^{s}=0 \in\left[M_{2^{u}}^{7}, M_{2^{u}}^{7}\right]$ for $s>1$, take the invertible transforma-

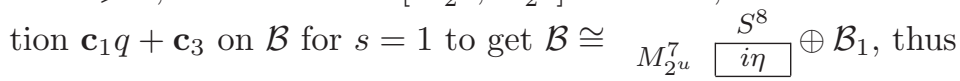

$$
\left(C_{u}^{5} \wedge C_{r}^{5, s}\right)^{(9)} \simeq \mathbf{C}_{\mathcal{B}} \simeq \mathbf{C}_{i \eta} \vee \mathbf{C}_{\mathcal{B}_{1}}=C_{u}^{9} \vee \mathbf{C}_{\mathcal{B}_{1}}
$$

Lemma 4.5. The mapping cone of the map $\begin{array}{ccc}C_{1}^{8,1} & M_{2}^{7} & M_{2}^{7} \\ & 1 \wedge \text { in } & i \eta \wedge 1\end{array}$ is homotopy equivalent to $M_{2}^{3} \wedge C_{\eta}^{5} \vee C_{1}^{9,1}$.

Proof. $\Sigma C_{1}^{5} \wedge C_{1}^{5,1} \simeq C_{1}^{5} \wedge M_{2}^{3} \wedge M_{2}^{1} \simeq\left(M_{2}^{3} \wedge C_{\eta}^{5} \vee M_{2}^{7}\right) \wedge M_{2}^{1} \simeq C_{1}^{6,1} \wedge C_{\eta}^{5} \vee$ $C_{1}^{10,1}$, hence $C_{1}^{5} \wedge C_{1}^{5,1} \simeq C_{1}^{5,1} \wedge C_{\eta}^{5} \vee C_{1}^{9,1}$. Together with $\left(C_{1}^{5,1} \wedge C_{\eta}^{5}\right)^{(9)} \simeq M_{2}^{3} \wedge$ $C_{\eta}^{5} \vee C_{1}^{9}$ (from Lemma 4.3), we have $\left(C_{1}^{5} \wedge C_{1}^{5,1}\right)^{(9)} \simeq M_{2}^{3} \wedge C_{\eta}^{5} \vee C_{1}^{9} \vee C_{1}^{9,1}$. On the other hand, from (12), $\left(C_{1}^{5} \wedge C_{1}^{5,1}\right)^{(9)} \simeq C_{1}^{9} \vee C_{1}^{8,1} \cup_{(1 \wedge i \eta, i \eta \wedge 1)} \mathbf{C}\left(M_{2}^{7} \vee M_{2}^{7}\right)$. So by Lemma 2.6, $C_{1}^{8,1} \cup_{(1 \wedge i \eta, i \eta \wedge 1)} \mathbf{C}\left(M_{2}^{7} \vee M_{2}^{7}\right) \simeq M_{2}^{3} \wedge C_{\eta}^{5} \vee C_{1}^{9,1}$.

Now, from (10), (11), (12) and Lemma 4.5 we get

$$
\begin{aligned}
& \left(C_{u}^{5} \wedge C_{r}^{5, s}\right)^{(9)} \simeq C_{u}^{9} \vee C_{u}^{9, r} \vee M_{2^{u}}^{3} \wedge C_{\eta}^{5} \quad(s \geqslant u \text { and } r \geqslant u) ; \\
& \left(C_{u}^{5} \wedge C_{r}^{5, s}\right)^{(9)} \simeq C_{u}^{9} \vee C_{r}^{9, u} \vee M_{2^{r}}^{3} \wedge C_{\eta}^{5} \quad(s \geqslant u>r) .
\end{aligned}
$$

- For $u>s$, it is easy to get

$$
\begin{aligned}
& \left(C_{u}^{5} \wedge C_{r}^{5, s}\right)^{(9)} \simeq M_{2^{r}}^{3} \wedge C_{\eta}^{5} \vee \mathbf{C}_{\mathcal{B}^{\prime}} \quad(u>s \text { and } u \geqslant r) \\
& \left(C_{u}^{5} \wedge C_{r}^{5, s}\right)^{(9)} \simeq \mathbf{C}_{\mathcal{B}^{\prime \prime}} \quad(r>u>s),
\end{aligned}
$$

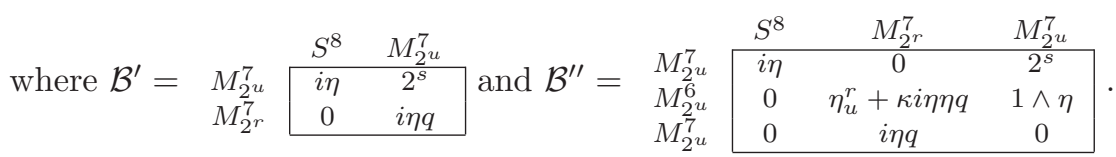

The decomposability of $C_{u}^{5} \wedge C_{r}^{5, s}$ can be obtained from the structure of $\left(C_{u}^{5} \wedge\right.$ $\left.C_{r}^{5, s}\right) / S^{6}$ and $\left(C_{u}^{5} \wedge C_{r}^{5, s}\right)^{(9)}$ now.

(i) For $s<u<r, C_{u}^{5} \wedge C_{r}^{5, s}$ is indecomposable, otherwise by Corollary 4.4 and (6), $C_{u}^{5} \wedge C_{r}^{5, s} \simeq X \vee C_{u}^{9, r}$. However, $\pi_{8} C_{u}^{9, r} \cong \mathbb{Z} / 2^{r+1}$ is not a direct summand of $\pi_{8}\left(C_{u}^{5} \wedge C_{r}^{5, s}\right) \cong\left[C^{5, u}, C_{r}^{5, s}\right] \cong \mathbb{Z} / 2^{s+1} \oplus \mathbb{Z} / 2^{r}$. 
(ii) For $u<s, u<r$, suppose $C_{u}^{5} \wedge C_{r}^{5, s}$ is decomposable, by Corollary 4.4 and (13), $C_{u}^{5} \wedge C_{r}^{5, s} \simeq X \vee C_{u}^{9, r}$. There is a cofibre sequence, $S^{7} \vee S^{8} \vee M_{2^{s}}^{7} \vee M_{2^{s}}^{8} \stackrel{\mathcal{A}^{\prime \prime}}{\longrightarrow}$ $S^{7} \vee S^{8} \vee S^{7} \vee M_{2^{s}}^{7} \rightarrow\left(C_{u}^{5} \wedge C_{r}^{5, s}\right) / S^{6} \rightarrow S^{8} \vee S^{9} \vee M_{2^{s}}^{8} \vee M_{2^{s}}^{9} \stackrel{\Sigma \mathcal{A}^{\prime \prime}}{\longrightarrow} S^{8} \vee S^{9} \vee$ $S^{8} \vee M_{2^{s}}^{8}$, where $\mathcal{A}^{\prime \prime}$ is the map in (9). So,

$$
\mathbb{Z} \oplus \mathbb{Z} / 2^{s} \stackrel{\left[\Sigma \mathcal{A}^{\prime \prime}, S^{9}\right]}{\longrightarrow} \mathbb{Z} \oplus \mathbb{Z} / 2^{s} \oplus \mathbb{Z} / 2 \rightarrow\left[\left(C_{u}^{5} \wedge C_{r}^{5, s}\right) / S^{6}, S^{9}\right] \rightarrow 0,
$$

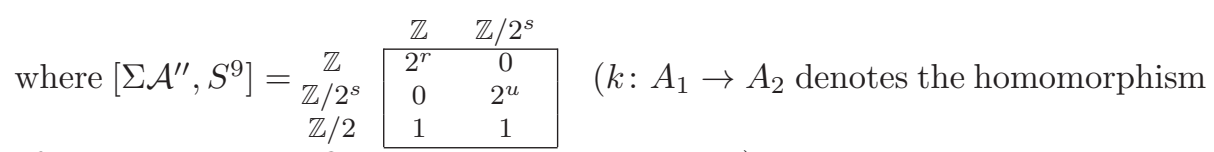
of abelian groups defined by multiplication by $k$ ). Thus $\left[C_{u}^{5} \wedge C_{r}^{5, s}, S^{9}\right] \cong\left[\left(C_{u}^{5} \wedge C_{r}^{5, s}\right) / S^{6}, S^{9}\right] \cong \frac{\mathbb{Z} \oplus \mathbb{Z} / 2^{s} \oplus \mathbb{Z} / 2}{\left\langle\left(2^{r}, 0,1\right),\left(0,2^{u}, 1\right)\right\rangle} \cong \mathbb{Z} / 2^{r+1} \oplus \mathbb{Z} / 2^{u}$, which is a contradiction since $\left[C_{u}^{9, r}, S^{9}\right] \cong \mathbb{Z} / 2^{r}$. Hence $C_{u}^{5} \wedge C_{r}^{5, s}$ is indecomposable for $u<s$ and $u<r$.

(iii) For $r \leqslant u<s$, the indecomposability of $C_{u}^{5} \wedge C_{r}^{5, s}$ is easily obtained from the following lemma.

Lemma 4.6. The wedge summand $\mathbf{C}_{\mathcal{A}^{\prime}}$ in (8) is indecomposable.

Proof. Assume that $\mathbf{C}_{\mathcal{A}^{\prime}} \simeq Z_{1} \vee Z_{2}$ is decomposable and $H_{9} Z_{1} \neq 0$. It follows from the homology groups of $\mathbf{C}_{\mathcal{A}^{\prime}}$ and $S q^{2}$ on $H^{*}\left(\mathbf{C}_{\mathcal{A}^{\prime}} ; \mathbb{Z} / 2\right)$ that $Z_{2}=C_{u}^{9, u}$ or $C_{r}^{9, u}$, which contradicts $\left[\mathbf{C}_{\mathcal{A}^{\prime}}, S^{9}\right] \cong \mathbb{Z} / 2^{u+1}$.

(iv) For $u \geqslant s, u \geqslant r$ or $u=s<r$.

Lemma 4.7. In (15), $\mathbf{C}_{\mathcal{B}^{\prime}} \simeq C_{r}^{9, s} \vee C_{s}^{9}$

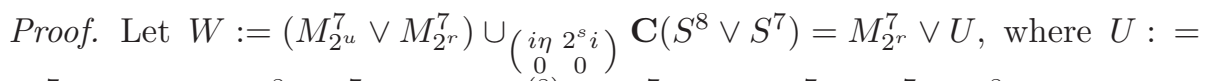
$M_{2^{u}}^{7} \cup_{\left(i \eta, 2^{s} i\right)} \mathbf{C}\left(S^{8} \vee S^{7}\right)$. From $U^{(8)}=M_{2^{u}}^{7} \cup_{2^{s} i} \mathbf{C} S^{7} \simeq M_{2^{s}}^{7} \vee S^{8}$ and $H_{8} U \cong$ $\pi_{8} U=\mathbb{Z}$ we get $U \simeq S^{8} \vee C_{s}^{9}, W \simeq M_{2^{r}}^{7} \vee S^{8} \vee C_{s}^{9}$.

There is a cofibre sequence

$$
S^{8} \stackrel{\left(\begin{array}{c}
y i \eta \\
b \\
0
\end{array}\right)}{\longrightarrow} W \simeq M_{2^{r}}^{7} \vee S^{8} \vee C_{s}^{9} \rightarrow \mathbf{C}_{\mathcal{B}^{\prime}} \rightarrow S^{9},
$$

for some $y \in\{0,1\}$ and $b \in \mathbb{Z}$. From $H_{8}\left(\mathbf{C}_{\mathcal{B}^{\prime}}\right)=\mathbb{Z} / 2^{s}, b=2^{s}$. Since $S q^{2}$ is isomorphic on both $H^{7}\left(\left(C_{u}^{5} \wedge C_{r}^{5, s}\right)^{(9)} ; \mathbb{Z} / 2\right)$ and $H^{7}\left(\mathbf{C}_{\mathcal{B}^{\prime}} ; \mathbb{Z} / 2\right), y=1$. Hence $\mathbf{C}_{\mathcal{B}^{\prime}} \simeq C_{r}^{9 s} \vee C_{s}^{9}$.

From (7), (14), (15), together with Lemma 4.7, for $u \geqslant s, u \geqslant r$,

$$
\begin{gathered}
\left(C_{u}^{5} \wedge C_{r}^{5, s}\right) / S^{6} \simeq C_{r}^{9, s} \vee C^{9, r} \vee M_{2^{s}}^{4} \wedge C_{\eta}^{5} \\
\left(C_{u}^{5} \wedge C_{r}^{5, s}\right)^{(9)} \simeq M_{2^{r}}^{3} \wedge C_{\eta}^{5} \vee C_{s}^{9} \vee C_{r}^{9, s}
\end{gathered}
$$

Now we can get the following decomposition from $\left(C_{u}^{5} \wedge C_{r}^{5, s}\right) / S^{6}$ and $\left(C_{u}^{5} \wedge\right.$ 
$\left.C_{r}^{5, s}\right)^{(9)}$

$$
C_{u}^{5} \wedge C_{r}^{5, s} \simeq\left\{\begin{array}{l}
C_{r}^{9, s} \vee C_{\eta}^{5} \wedge C_{r}^{5, s}, \quad u \geqslant r \text { and } u \geqslant s, \\
C_{s}^{9, r} \vee C_{\eta}^{5} \wedge C_{r}^{5, r}, \quad u=r<s .
\end{array}\right.
$$

The proof, which we will omit, is similar to the proof of the case $C_{r}^{5, s} \wedge C_{r^{\prime}}^{5, s^{\prime}}(s=$ $\left.r^{\prime}>s^{\prime}>r\right)$, given in the next section. For details, see our preprint [17].

\section{Decomposition of $C_{r}^{5, s} \wedge C_{r^{\prime}}^{5, s^{\prime}}, r, r^{\prime}, s, s^{\prime} \in \mathbb{Z}^{+}$}

\subsection{Preliminaries}

Similar to Corollary 4.4 we have

Lemma 5.1. If $C_{r}^{5, s} \wedge C_{r^{\prime}}^{5, s^{\prime}}$ is decomposable, then $C_{r}^{5, s} \wedge C_{r^{\prime}}^{5, s^{\prime}} \simeq X \vee C_{l}^{9, k}$ or $C_{r}^{5, s} \wedge$ $C_{r^{\prime}}^{5, s^{\prime}} \simeq X \vee C_{l}^{9, k} \vee C_{l^{\prime}}^{9, k^{\prime}}$, where $X$ is indecomposable, $H_{6} X \neq 0$ and $\left\{k, k^{\prime}\right\} \subset$ $\left\{m_{r, s^{\prime}}, m_{s, r^{\prime}}, m_{r, r^{\prime}}\right\},\left\{l, l^{\prime}\right\} \subset\left\{m_{s, s^{\prime}}, m_{r, s^{\prime}}, m_{s, r^{\prime}}\right\}$.

By $C_{r}^{5, s} \wedge C_{r^{\prime}}^{5, s^{\prime}} \simeq D\left(C_{s}^{5, r} \wedge C_{s^{\prime}}^{5, r^{\prime}}\right)$, we can assume $s=\max \left\{r, s, r^{\prime}, s^{\prime}\right\}$.

Lemma 5.2. If $s>r^{\prime}, s^{\prime}$, then $C_{r}^{5, s} \wedge C_{r^{\prime}}^{5, s^{\prime}} \simeq C_{r^{\prime}}^{9, s^{\prime}} \vee C_{r}^{5} \wedge C_{r^{\prime}}^{5, s^{\prime}}$, hence

$$
C_{r}^{5, s} \wedge C_{r^{\prime}}^{5, s^{\prime}} \simeq \begin{cases}C_{r^{\prime}}^{9, s^{\prime}} \vee C_{r^{\prime}}^{9, s^{\prime}} \vee C_{\eta}^{5} \wedge C_{r^{\prime}}^{5, s^{\prime}}, & s>r^{\prime}, s^{\prime} \text { and } r \geqslant r^{\prime}, s^{\prime}, \\ C_{r^{\prime}}^{9, s^{\prime}} \vee C_{s^{\prime}}^{9, r^{\prime}} \vee C_{\eta}^{5} \wedge C_{r}^{5, r}, & s>r^{\prime}>s^{\prime}=r, \\ C_{r^{\prime}}^{9, s^{\prime}} \vee C_{r}^{5} \wedge C_{r^{\prime}}^{5, s^{\prime}}, & s>r^{\prime}, s^{\prime} \text { and } s^{\prime} \neq r<r^{\prime} \text { or } r<s^{\prime} .\end{cases}
$$

Proof. Since $\left|\left[C_{r^{\prime}}^{5, s^{\prime}}, C_{r^{\prime}}^{5, s^{\prime}}\right]\right|=\max \left\{2^{s^{\prime}+1}, 2^{r^{\prime}+1}\right\}[\mathbf{1}]$, this lemma is easily obtained from the cofibre sequence $S^{4} \wedge C_{r^{\prime}}^{5, s^{\prime}} \stackrel{\left(\begin{array}{c}i \eta \\ 2^{s}\end{array}\right) \wedge 1}{\longrightarrow}\left(M_{2^{r}}^{3} \vee S^{4}\right) \wedge C_{r^{\prime}}^{5, s^{\prime}} \rightarrow C_{r}^{5, s} \wedge C_{r^{\prime}}^{5, s^{\prime}}$.

Now by $C_{r}^{5, s} \wedge C_{r^{\prime}}^{5, s^{\prime}} \simeq C_{r^{\prime}}^{5, s^{\prime}} \wedge C_{r}^{5, s}$ and $C_{r}^{5, s} \wedge C_{r^{\prime}}^{5, s^{\prime}} \simeq D\left(C_{s}^{5, r} \wedge C_{s^{\prime}}^{5, r^{\prime}}\right)$, it suffices to compute the following cases, denoted by Cases
(a) $s=r^{\prime}>s^{\prime}>r$
(b) $s=r^{\prime}>s^{\prime}=r$;
(c) $s=r^{\prime}=s^{\prime}>r$;
(d) $s=r^{\prime}=s^{\prime}=r$;
(e) $s=s^{\prime}>r^{\prime}>r$;
(f) $s=s^{\prime}>r^{\prime}=r$.

We will prove case (a) of Cases and omit the proofs of the other cases since they are similar or easier.

5.2. $\left(C_{r}^{5, s} \wedge C_{r^{\prime}}^{5, s^{\prime}}\right) / S^{6}$ and $\left(C_{r}^{5, s} \wedge C_{r^{\prime}}^{5, s^{\prime}}\right)^{(9)}$ for $s=r^{\prime}>s^{\prime}>r$

(1) Determining $\left(C_{r}^{5, s} \wedge C_{r^{\prime}}^{5, s^{\prime}}\right) / S^{6}$

Applying Lemma 2.4 to the cofibre sequences

$$
S_{1}^{3} \vee M_{2^{s}}^{3} \stackrel{f=\left(2^{r}, \eta q\right)}{\longrightarrow} S_{a}^{3} \rightarrow C_{r}^{5, s} ; \quad S_{2}^{3} \vee M_{2^{s^{\prime}}}^{3} \stackrel{f^{\prime}=\left(2^{r^{\prime}}, \eta q\right)}{\longrightarrow} S_{b}^{3} \rightarrow C_{r^{\prime}}^{5, s^{\prime}},
$$

with the identification $\Sigma M_{2^{s}}^{3} \wedge M_{2^{s^{\prime}}}^{3} \simeq M_{2^{s^{\prime}}}^{7} \vee M_{2^{s^{\prime}}}^{8}$ we have

$$
\begin{aligned}
& \left(C_{r}^{5, s} \wedge C_{r^{\prime}}^{5, s^{\prime}}\right) / S^{6} \simeq\left(S^{7} \vee M_{2^{s}}^{7} \vee S^{7} \vee M_{2^{s^{\prime}}}^{7}\right) \cup \mathcal{A} \mathbf{C}\left(S^{7} \vee M_{2^{s^{\prime}}}^{7} \vee M_{2^{s}}^{7} \vee M_{2^{s^{\prime}}}^{7} \vee M_{2^{s^{\prime}}}^{8}\right),
\end{aligned}
$$

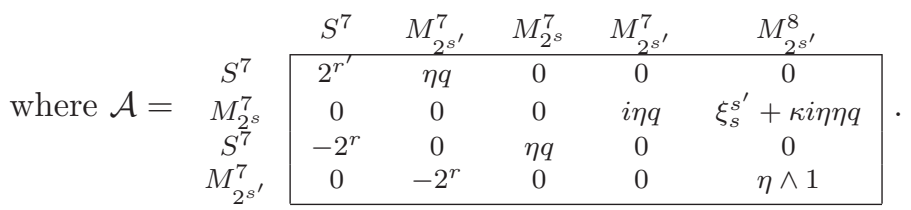


Take transformations $2^{r^{\prime}-r} \mathbf{r}_{3}+\mathbf{r}_{1} ;-\mathbf{r}_{3} ;-\mathbf{r}_{4}$ on $\mathcal{A}$ to get $\left(C_{r}^{5, s} \wedge C_{r^{\prime}}^{5, s^{\prime}}\right) / S^{6} \simeq$ $C_{r}^{9, s} \vee L$, where $L$ is the mapping cone of the map

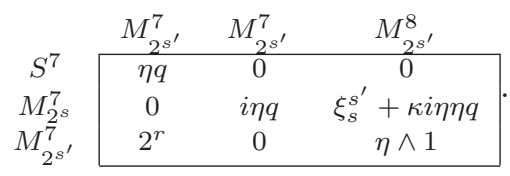

Lemma 5.3. $L \simeq C^{9, r} \vee\left(C_{s}^{9, s^{\prime}} \vee C_{r}^{9}\right) \cup\left(\begin{array}{c}\alpha \\ \gamma\end{array}\right)$ C $S^{9}$ where $\alpha=i_{\underline{M}}\left(\begin{array}{c}\xi_{s} \\ 0\end{array}\right)$ and $\gamma$ is determined by $q_{S} \gamma=\left(\begin{array}{c}0 \\ 2^{s^{\prime}}\end{array}\right)\left(i_{\underline{M}}\right.$ and $q_{S}$ are the maps in Cof4 of $C_{s}^{9, s^{\prime}}$ and Cof1 of $C_{r}^{9}$, respectively).

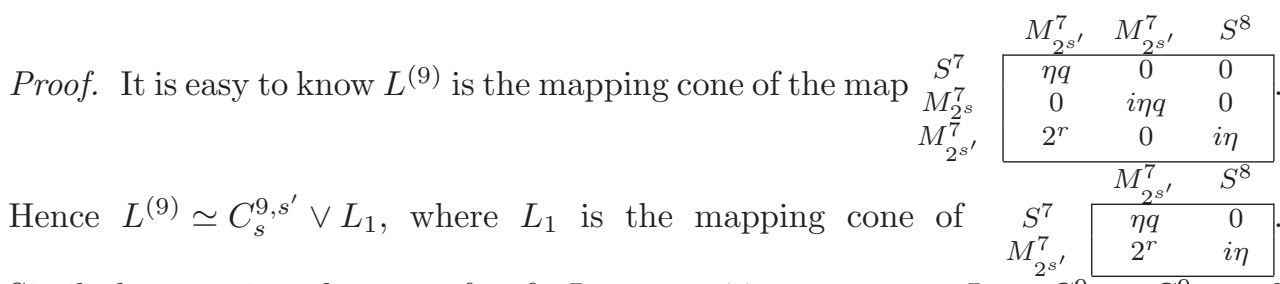
Similarly as in the proof of Lemma 4.7, we get $L_{1} \simeq C^{9, r} \vee C_{r}^{9}$ and $L^{(9)} \simeq C_{s}^{9, s^{\prime}} \vee C^{9, r} \vee C_{r}^{9}$.

There is a cofibre sequence

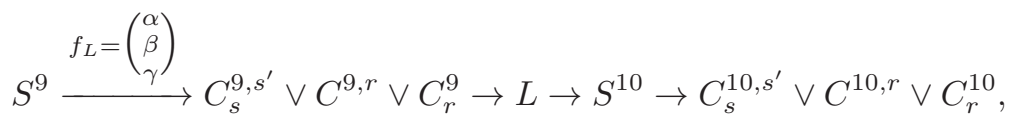

$\alpha=i_{\underline{M}}\left(\begin{array}{c}z \xi_{s} \\ x \eta\end{array}\right), \beta=i_{S}\left(\begin{array}{c}0 \\ y \eta\end{array}\right), \gamma$ is determined by $q_{S} \gamma=\left(\begin{array}{c}w \eta \\ a\end{array}\right)$, where $x, y, z, w \in\{0,1\}$ and $a \in \mathbb{Z}$.
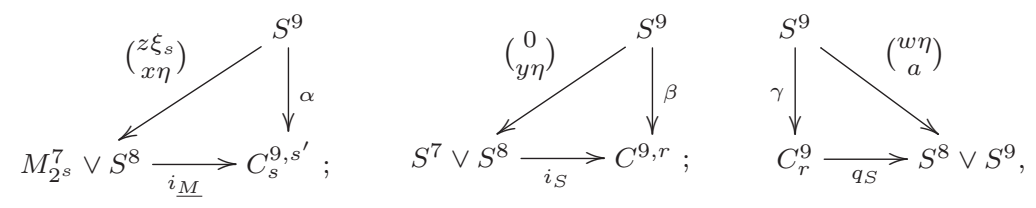

$a=2^{s^{\prime}}$ from $\left[L, S^{10}\right] \cong \mathbb{Z} / 2^{s^{\prime}}$. From Proposition 2.1, for $s=r^{\prime}>s^{\prime}>r$, $\left[\left(C_{r}^{5, s} \wedge C_{r^{\prime}}^{5, s^{\prime}}\right) / S^{6}, S^{8}\right] \cong\left[C_{r}^{5, s} \wedge C_{r^{\prime}}^{5, s^{\prime}}, S^{8}\right] \cong\left[C_{r}^{5, s}, C_{s^{\prime}}^{5, r^{\prime}}\right] \cong \mathbb{Z} / 2^{s} \oplus \mathbb{Z} / 2^{r+1} \oplus \mathbb{Z} / 2^{r+1}$.

Together with $\left[\left(C_{r}^{5, s} \wedge C_{r^{\prime}}^{5, s^{\prime}}\right) / S^{6}, S^{8}\right] \cong\left[C_{r}^{9, s}, S^{8}\right] \oplus\left[L, S^{8}\right]$, we get

$$
\left[L, S^{8}\right] \cong \mathbb{Z} / 2^{s} \oplus \mathbb{Z} / 2^{r+1} .
$$

On the other hand, by the cofibre sequence of $L$ above, there is an exact sequence

$$
0 \rightarrow \frac{\mathbb{Z} / 2}{\langle x, y, z, w\rangle} \rightarrow\left[L, S^{8}\right] \rightarrow \mathbb{Z} / 2^{s+1} \oplus \mathbb{Z} / 2^{r+1} \stackrel{\left(\alpha^{*}, \gamma^{*}\right)}{\longrightarrow} \mathbb{Z} / 2,
$$


where $\alpha^{*}(1)=z, \gamma^{*}(1)=w$. Hence $w=0, z=1$. By the following commutative diagram:

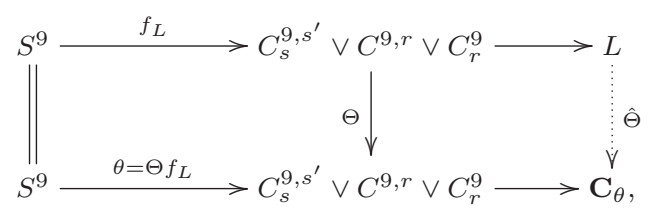

$\begin{array}{c|ccc}\multicolumn{1}{c}{} & C_{s}^{9, s^{\prime}} & C^{9, r} & C_{r}^{9} \\$\cline { 2 - 4 } \text {$\left.where } \Theta=C_{s}^{9, s^{\prime}} & \hat{\mu} & 0 & 0 \\ C^{9, r} & \hat{\lambda} & 1 & 0 \\ C_{r}^{9} & 0 & 0 & 1\end{array}\right]$ is a homotopy equivalence, $\hat{\mu}$ and $\hat{\lambda}$ are induced by the following commutative diagrams:
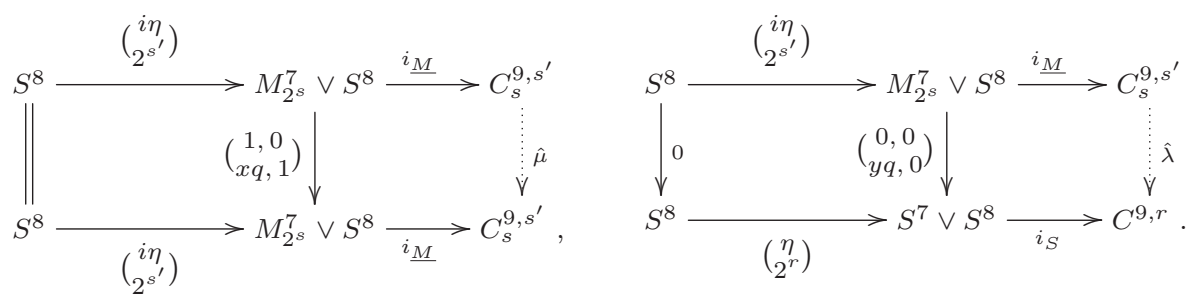

Then $\theta=\Theta f_{L}=\left(\begin{array}{c}\hat{\mu} \alpha \\ \gamma \\ \gamma\end{array}\right)=\left(\begin{array}{c}\hat{\mu} \alpha \\ 0 \\ \gamma\end{array}\right)$. Rewrite $\hat{\mu} \alpha$ as $\alpha$,

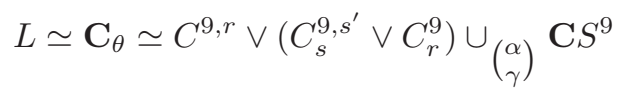

$\alpha, \gamma$ satisfy the conditions in the lemma.

Thus

$$
\left(C_{r}^{5, s} \wedge C_{r^{\prime}}^{5, s^{\prime}}\right) / S^{6} \simeq C_{r}^{9, s} \vee C^{9, r} \vee\left(C_{s}^{9, s^{\prime}} \vee C_{r}^{9}\right) \cup_{\left(\begin{array}{c}
\alpha \\
\gamma
\end{array}\right)} \mathbf{C} S^{9}, s=r^{\prime}>s^{\prime}>r .
$$

(2) Determining $\left(C_{r}^{5, s} \wedge C_{r^{\prime}}^{5, s^{\prime}}\right)^{(9)}$

Apply Lemma 2.4 to Cof4 of $C_{r}^{5, s}$ and $C_{r^{\prime}}^{5, s^{\prime}}$ to get

$$
\begin{aligned}
& \left(C_{r}^{5, s} \wedge C_{r^{\prime}}^{5, s^{\prime}}\right)^{(9)} \simeq\left(M_{2^{r}}^{3} \wedge M_{2^{r^{\prime}}}^{3} \vee M_{2^{r}}^{7} \vee M_{2^{r^{\prime}}}^{7} \vee S^{8}\right) \cup_{\mathcal{B}} \mathbf{C}\left(M_{2^{r}}^{7} \vee S^{8} \vee M_{2^{r^{\prime}}}^{7} \vee S^{8}\right),
\end{aligned}
$$

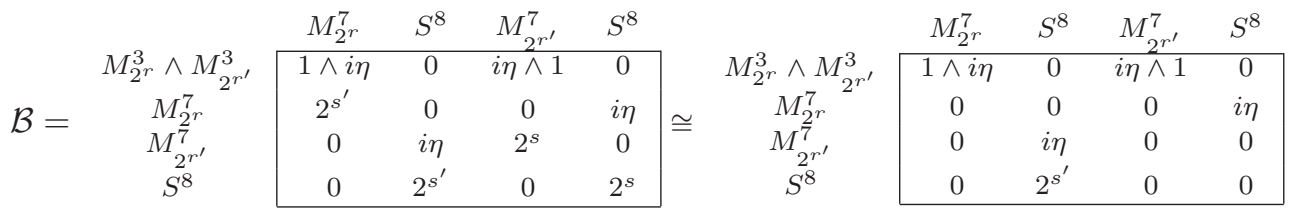

by noting that $2^{s^{\prime}}=0 \in\left[M_{2^{r}}^{7}, M_{2^{r}}^{7}\right]$ and $2^{s}=0 \in\left[M_{2^{r^{\prime}}}^{7}, M_{2^{r^{\prime}}}^{7}\right]$ for $s=r^{\prime}>s^{\prime}>r$. From (11), for $r^{\prime}>r,\left(M_{2^{r}}^{3} \wedge M_{2^{r^{\prime}}}^{3}\right) \cup_{(1 \wedge i \eta, i \eta \wedge 1)} \mathbf{C}\left(M_{2^{r}}^{7} \vee M_{2^{r^{\prime}}}^{7}\right) \simeq M_{2^{r}}^{3} \wedge C_{\eta}^{5} \vee C_{r}^{9, r^{\prime}}$. Thus

$$
\left(C_{r}^{5, s} \wedge C_{r^{\prime}}^{5, s^{\prime}}\right)^{(9)} \simeq M_{2^{r}}^{3} \wedge C_{\eta}^{5} \vee C_{r}^{9, r^{\prime}} \vee C_{r^{\prime}}^{9, s^{\prime}} \vee C_{r}^{9} \quad \text { for } s=r^{\prime}>s^{\prime}>r
$$




\subsection{Decomposition of $C_{r}^{5, s} \wedge C_{r^{\prime}}^{5, s^{\prime}}$ for $s=r^{\prime}>s^{\prime}>r$}

Denote a column vector by $\left(\zeta_{1}, \zeta_{2}, \cdots, \zeta_{s}\right)^{T}$ in the rest of the paper.

From (17), there is a cofibre sequence

$$
S^{9} \stackrel{\left(\delta_{1}, \mu, \nu, \omega\right)^{T}}{\longrightarrow} M_{2^{r}}^{3} \wedge C_{\eta}^{5} \vee C_{r}^{9, s} \vee C_{r^{\prime}}^{9, s^{\prime}} \vee C_{r}^{9} \rightarrow C_{r}^{5, s} \wedge C_{r^{\prime}}^{5, s^{\prime}}
$$

Since $\delta_{1}=i_{\bar{C}} t_{1} \varrho_{6}$ for some $t_{1} \in \mathbb{Z}$,

$$
\left(C_{r}^{5, s} \wedge C_{r^{\prime}}^{5, s^{\prime}}\right) / S^{6} \simeq C^{9, r} \vee\left(C_{r}^{9, s} \vee C_{r^{\prime}}^{9, s^{\prime}} \vee C_{r}^{9}\right) \cup_{(\mu, \nu, \omega)^{T}} \mathbf{C} S^{9}
$$

On the other hand, from (16)

$$
\left(C_{r}^{5, s} \wedge C_{r^{\prime}}^{5, s^{\prime}}\right) / S^{6} \simeq C^{9, r} \vee\left(C_{r}^{9, s} \vee C_{r^{\prime}}^{9, s^{\prime}} \vee C_{r}^{9}\right) \cup_{(0, \alpha, \gamma)^{T}} \mathbf{C} S^{9}
$$

Thus $\left(C_{r}^{9, s} \vee C_{r^{\prime}}^{9, s^{\prime}} \vee C_{r}^{9}\right) \cup_{(\mu, \nu, \omega)^{T}} \mathbf{C} S^{9} \simeq\left(C_{r}^{9, s} \vee C_{r^{\prime}}^{9, s^{\prime}} \vee C_{r}^{9}\right) \cup_{(0, \alpha, \gamma)^{T}} \mathbf{C} S^{9}$, which are 2 -local spaces. Consequently, there is a homotopy equivalence $\lambda$ yielding the following homotopy commutative diagram:

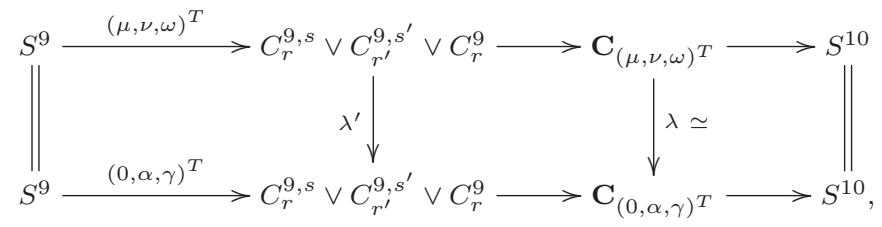

where $\lambda^{\prime}$ is the restriction of $\lambda$, which is a self-homotopy equivalence of $C_{r}^{9, s} \vee C_{r^{\prime}}^{9, s^{\prime}} \vee$ $C_{r}^{9}$.

Let $\widetilde{\Gamma}$ be induced by the following commutative diagram:

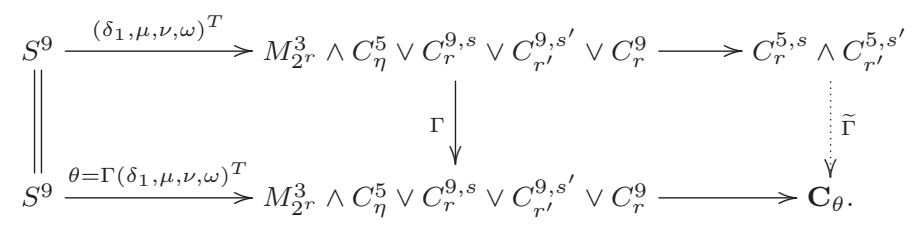

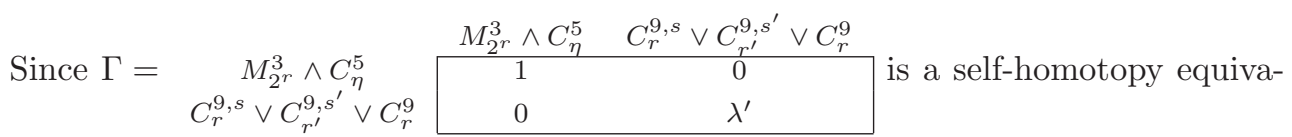
lence, $\widetilde{\Gamma}$ is also a homotopy equivalence.

$$
\theta=\Gamma\left(\delta_{1}, \mu, \nu, \omega\right)^{T}=\left(\delta_{1}, \lambda^{\prime}(\mu, \nu, \omega)^{T}\right)^{T}=\left(\delta_{1}, 0, \alpha, \gamma\right)^{T} .
$$

Consequently, for $s=r^{\prime}>s^{\prime}>r$,

$$
C_{r}^{5, s} \wedge C_{r^{\prime}}^{5, s^{\prime}} \simeq \mathbf{C}_{\theta} \simeq C_{r}^{9, s} \vee Q_{1}, \quad Q_{1}=\left(M_{2^{r}}^{3} \wedge C_{\eta}^{5} \vee C_{r^{\prime}}^{9, s^{\prime}} \vee C_{r}^{9}\right) \cup_{\left(\delta_{1}, \alpha, \gamma\right)^{T}} \mathbf{C} S^{9} .
$$

Lemma 5.4. $Q_{1} \simeq C^{5, s^{\prime}} \wedge C_{r}^{5, s}, s=r^{\prime}>s^{\prime}>r$.

Proof. (1) Determining $\delta_{1}$, i.e., $t_{1}$

By Lemma 5.1, $C_{r}^{5, s} \wedge C_{r^{\prime}}^{5, s^{\prime}}$ cannot split out $M_{2^{r}}^{3} \wedge C_{\eta}^{5}$, which implies that $\delta_{1} \neq 0$ in $\pi_{9}\left(M_{2^{r}}^{3} \wedge C_{\eta}^{5}\right)$. Hence we can assume that $t_{1}=1$ for $r=1$ and $t_{1} \in\{1,2\}$ for $r \geqslant 2$. Next we determine $t_{1}$ for $r \geqslant 2$. 
Lemma 5.5. For $s \geqslant r^{\prime}, s^{\prime}$ and $r \geqslant 2$, there is a short exact sequence of abelian groups

$$
0 \rightarrow \mathbb{Z} / 2 \oplus \mathbb{Z} / 2 \oplus \mathbb{Z} / 2 \oplus \mathbb{Z} / 2^{s^{\prime}+\epsilon_{r^{\prime}}} \rightarrow \pi_{9}\left(C_{r}^{5, s} \wedge C_{r^{\prime}}^{5, s^{\prime}}\right) \rightarrow \mathbb{Z} / 2 \oplus \mathbb{Z} / 2 \rightarrow 0,
$$

where $\epsilon_{r^{\prime}}=0$ for $r^{\prime}=1$ and $\epsilon_{r^{\prime}}=1$ for $r^{\prime}>1$.

Proof. We only prove the case $r^{\prime}>1$

$$
\pi_{9}\left(C_{r}^{5, s} \wedge C_{r^{\prime}}^{5, s^{\prime}}\right) \cong\left[C_{s}^{5, r} \wedge C_{s^{\prime}}^{5, r^{\prime}}, S^{7}\right] \cong\left[C_{s}^{7, r}, C_{r^{\prime}}^{6, s^{\prime}}\right] \quad \text { (Proposition 2.1). }
$$

By Cof5 of $C_{s}^{7, r}$, there is an exact sequence

$$
\left[S^{7}, C_{r^{\prime}}^{6, s^{\prime}}\right] \stackrel{\left(2^{r} p_{1} q_{S}\right)^{*}}{\longrightarrow}\left[C_{s}^{7}, C_{r^{\prime}}^{6, s^{\prime}}\right] \rightarrow\left[C_{s}^{7, r}, C_{r^{\prime}}^{6, s^{\prime}}\right] \rightarrow\left[S^{6}, C_{r^{\prime}}^{6, s^{\prime}}\right] \stackrel{0}{\longrightarrow}\left[C_{s}^{7}, C_{r^{\prime}}^{6, s^{\prime}}\right] .
$$

$\left(2^{r} p_{1} q_{S}\right)^{*}$ is zero for $r \geqslant 2$ and $\left[C_{s}^{7}, C_{r^{\prime}}^{6, s^{\prime}}\right] \cong\left[S^{9}, C^{5, s} \wedge C_{r^{\prime}}^{5, s^{\prime}}\right] \cong \pi_{9}\left(C_{r^{\prime}}^{9, s^{\prime}} \vee C_{\eta}^{5} \wedge C_{r^{\prime}}^{5, s^{\prime}}\right)$ $\cong \mathbb{Z} / 2 \oplus \mathbb{Z} / 2 \oplus \mathbb{Z} / 2 \oplus \mathbb{Z} / 2^{s^{\prime}+1}$ for $s \geqslant r^{\prime}, s^{\prime}$ (the last isomorphism is from (4)).

There is a cofibre sequence

$$
S^{9} \stackrel{\left(\delta_{1}, \alpha, \gamma\right)^{T}}{\longrightarrow} M_{2^{r}}^{3} \wedge C_{\eta}^{5} \vee C_{r^{\prime}}^{9, s^{\prime}} \vee C_{r}^{9} \rightarrow Q_{1} \rightarrow S^{10},
$$

which implies the following exact sequence

$$
\begin{aligned}
& \mathbb{Z} \stackrel{\left(\delta_{1 *}, \alpha_{*}, \gamma_{*}\right)^{T}}{\longrightarrow} \mathbb{Z} / 4\left\langle i_{\bar{C}} \varrho_{6}\right\rangle \oplus \mathbb{Z} / 2\left\langle i_{\underline{M}} j_{1} \xi_{r^{\prime}}\right\rangle \oplus \mathbb{Z} / 2\left\langle i_{\underline{M}} j_{2} \eta\right\rangle \oplus \mathbb{Z} / 2\left\langle\left(j_{1} \eta\right)_{S}^{-}\right\rangle \oplus \mathbb{Z}\left\langle\left(2 j_{2}\right)_{S}^{-}\right\rangle \\
& \longrightarrow \pi_{9} Q_{1} \longrightarrow 0,
\end{aligned}
$$

where $\delta_{1 *}(1)=t_{1} i_{\bar{C}} \varrho_{6}, \alpha_{*}(1)=i_{\underline{M}} j_{1} \xi_{r^{\prime}}$ and $\gamma_{*}(1)=2^{s^{\prime}-1}\left(2 j_{2}\right)_{S}^{-}$.

$$
\pi_{9} Q_{1} \cong \frac{\mathbb{Z} / 4 \oplus \mathbb{Z} / 2 \oplus \mathbb{Z} / 2 \oplus \mathbb{Z} / 2 \oplus \mathbb{Z}}{\left\langle\left(t_{1}, 1,0,0,2^{s^{\prime}-1}\right)\right\rangle} \cong \begin{cases}\mathbb{Z} / 2 \oplus \mathbb{Z} / 2 \oplus \mathbb{Z} / 2 \oplus \mathbb{Z} / 2^{s^{\prime}+1}, & t_{1}=1, \\ \mathbb{Z} / 4 \oplus \mathbb{Z} / 2 \oplus \mathbb{Z} / 2 \oplus \mathbb{Z} / 2^{s^{\prime}}, & t_{1}=2 .\end{cases}
$$

Since $\pi_{9}\left(C_{r}^{5, s} \wedge C_{r^{\prime}}^{5, s^{\prime}}\right) \cong \mathbb{Z} / 2 \oplus \mathbb{Z} / 2 \oplus \pi_{9} Q_{1}$ induced by $C_{r}^{5, s} \wedge C_{r^{\prime}}^{5, s^{\prime}} \simeq C_{r}^{9, s} \vee Q_{1}$ for $s=r^{\prime}>s^{\prime}>r$, together with the short exact sequence for $\pi_{9}\left(C_{r}^{5, s} \wedge C_{r^{\prime}}^{5, s^{\prime}}\right)$ in Lemma 5.5, we have

$$
\pi_{9} Q_{1}=\mathbb{Z} / 2 \oplus \mathbb{Z} / 2 \oplus \mathbb{Z} / 2 \oplus \mathbb{Z} / 2^{s^{\prime}+1}, \quad \text { i.e., } t_{1}=1, \quad \delta_{1}=i_{\bar{C}} \varrho_{6} .
$$

(2) The cell structure of $C^{5, s^{\prime}} \wedge C_{r}^{5, s}$

Apply Lemma 2.4 to Cof1 of $C^{5, s^{\prime}}$ and Cof4 of $C_{r}^{5, s}$ to get

$$
\left(C^{5, s^{\prime}} \wedge C_{r}^{5, s}\right)^{(9)} \simeq M_{2^{r}}^{3} \wedge C_{\eta}^{5} \vee C_{r^{\prime}}^{5, s^{\prime}} \vee C_{r}^{9}
$$

There is a cofibre sequence

$$
S^{9} \stackrel{(\hat{\delta}, \hat{\alpha}, \hat{\gamma})^{T}}{\longrightarrow} M_{2^{r}}^{3} \wedge C_{\eta}^{5} \vee C_{r^{\prime}}^{9, s^{\prime}} \vee C_{r}^{9} \rightarrow C^{5, s^{\prime}} \wedge C_{r}^{5, s} \rightarrow S^{10},
$$

where $\hat{\delta}=i_{\bar{C}} \hat{t} \varrho_{6}, \hat{\alpha}=i_{\underline{M}}\left(\begin{array}{c}\hat{x} \xi_{r^{\prime}} \\ \hat{y} \eta\end{array}\right)$ and $\hat{\gamma}$ is determined by $q_{S}\left(\begin{array}{c}\hat{z} \eta \\ \hat{a}\end{array}\right)$ for $\hat{x}, \hat{y}, \hat{z} \in\{0,1\}$ and $\hat{t}, \hat{a} \in \mathbb{Z}$, i.e., 

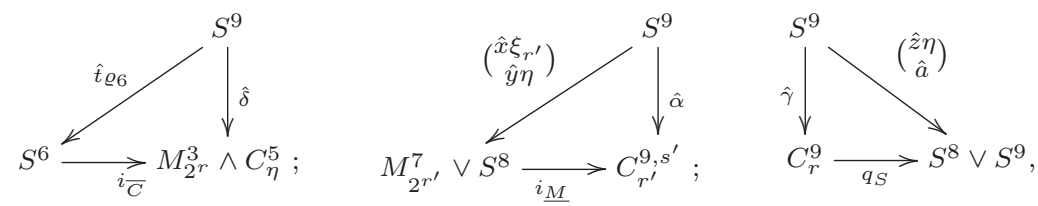

$\hat{a}=2^{s^{\prime}}$ for $H_{9}\left(C^{5, s^{\prime}} \wedge C_{r}^{5, s}\right) \cong \mathbb{Z} / 2^{s^{\prime}}$. Cofibre sequence (18) induces

$$
0 \rightarrow \frac{\mathbb{Z} / 2}{\langle\hat{x}, \hat{y}, \hat{z}\rangle} \rightarrow\left[C^{5, s^{\prime}} \wedge C_{r}^{5, s}, S^{8}\right] \rightarrow \mathbb{Z} / 2^{r^{\prime}+1} \oplus \mathbb{Z} / 2^{r+1} \stackrel{\left(\hat{\alpha}^{*}, \hat{\gamma}^{*}\right)}{\longrightarrow} \mathbb{Z} / 2,
$$

where $\hat{\alpha}^{*}(1)=\hat{x}$ and $\hat{\gamma}^{*}(1)=\hat{z}$. Since $C^{5, s^{\prime}} \wedge C_{r}^{5, s}$ is indecomposable for $s=r^{\prime}>s^{\prime}>$ $r, \hat{x}=1$ or $\hat{y}=1$, which implies $\frac{\mathbb{Z} / 2}{\langle\hat{x}, \hat{y}, \hat{z}\rangle}=0$. On the other hand,

$$
\left[C^{5, s^{\prime}} \wedge C_{r}^{5, s}, S^{8}\right] \cong\left[C_{r}^{5, s}, C_{s^{\prime}}^{5}\right] \cong \mathbb{Z} / 2^{r+1} \oplus \mathbb{Z} / 2^{r^{\prime}}
$$

Thus $\hat{x}=1$ and $\hat{z}=0$. Now from the following commutative diagram:

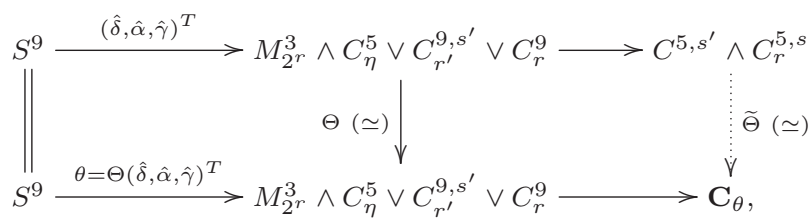

where the self-homotopy equivalence $\Theta$ is given by
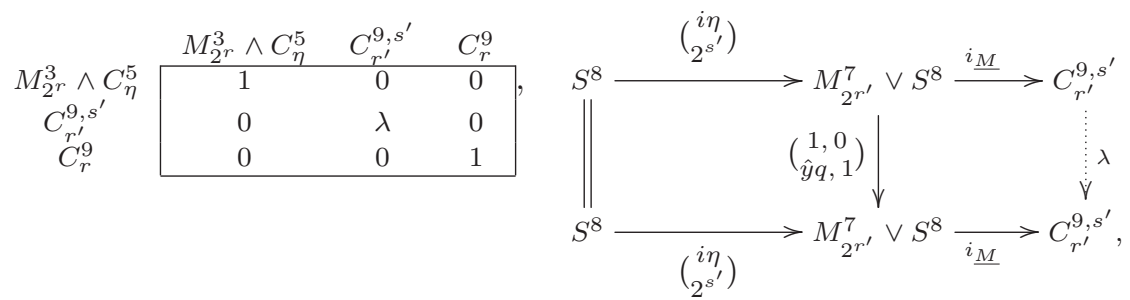

we can assume $\hat{y}=0$. Next we are going to calculate $\hat{\delta}$, i.e., $\hat{t}$.

$\hat{t}=1$ for $r=1$ and $\hat{t} \in\{1,2\}$ for $r \geqslant 2$ since $\hat{\delta} \neq 0$.

For the case $r \geqslant 2$, similarly as the calculation of $\pi_{9} Q_{1}$, we get

$$
\pi_{9}\left(C^{5, s^{\prime}} \wedge C_{r}^{5, s}\right) \cong \begin{cases}\mathbb{Z} / 2 \oplus \mathbb{Z} / 2 \oplus \mathbb{Z} / 2 \oplus \mathbb{Z} / 2^{s^{\prime}+1}, & \hat{t}=1, \\ \mathbb{Z} / 4 \oplus \mathbb{Z} / 2 \oplus \mathbb{Z} / 2 \oplus \mathbb{Z} / 2^{s^{\prime}}, & \hat{t}=2 .\end{cases}
$$

On the other hand, $\pi_{9}\left(C^{5, s^{\prime}} \wedge C_{r}^{5, s}\right) \cong\left[C_{s^{\prime}}^{7}, C_{r}^{6, s}\right]$ and from Cof3 of $C_{s^{\prime}}^{7}$, we get the following exact sequence

$$
\left[C_{\eta}^{8}, C_{r}^{6, s}\right] \stackrel{\left(i_{\eta} 2^{s^{\prime}}\right)^{*}=0}{\longrightarrow}\left[S^{6}, C_{r}^{6, s}\right] \rightarrow\left[C_{s^{\prime}}^{7}, C_{r}^{6, s}\right] \rightarrow\left[C_{\eta}^{7}, C_{r}^{6, s}\right] \stackrel{\left(i_{\eta} 2^{s^{\prime}}\right)^{*}}{\longrightarrow}\left[S^{5}, C_{r}^{6, s}\right] .
$$


There is a commutative diagram for the morphism $\left(i_{\eta} 2^{s^{\prime}}\right) *$ by $(3)$

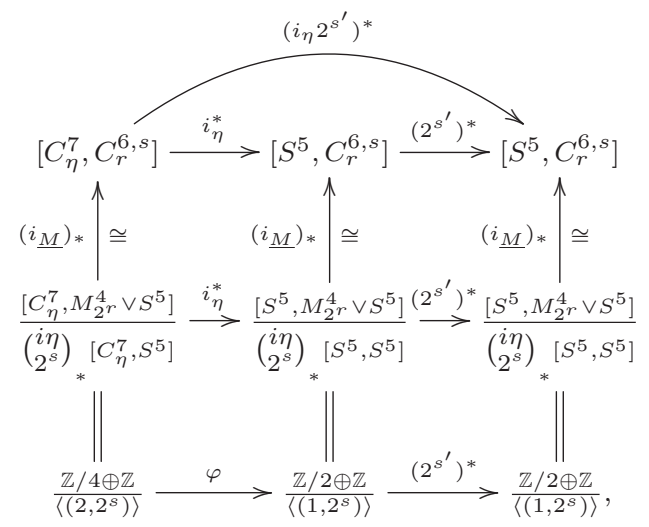

where $\varphi(1,0)=0$ and $\varphi(0,1)=(0,2)$. Hence $\operatorname{ker}\left(i_{\eta} 2^{s^{\prime}}\right)^{*} \cong \mathbb{Z} / 2 \oplus \mathbb{Z} / 2^{s^{\prime}+1}$ in $(20)$. Together with (19), we have

$$
\pi_{9}\left(C^{5, s^{\prime}} \wedge C_{r}^{5, s}\right) \cong \mathbb{Z} / 2 \oplus \mathbb{Z} / 2 \oplus \mathbb{Z} / 2 \oplus \mathbb{Z} / 2^{s^{\prime}+1}, \quad \text { i.e., } \hat{t}=1 .
$$

From the analysis above, we get $\hat{\delta}=\delta, \hat{\alpha}=\alpha$ and $\hat{\gamma}=\gamma$, i.e., $Q_{1} \simeq C^{5, s^{\prime}} \wedge C_{r}^{5, s}$.

Hence $C_{r}^{5, s} \wedge C_{r^{\prime}}^{5, s^{\prime}} \simeq C_{r}^{9, s} \vee C^{5, s^{\prime}} \wedge C_{r}^{5, s}$ for $s=r^{\prime}>s^{\prime}>r$.

\section{Acknowledgments}

This work was supported by National Natural Science Foundation of China (Grant Nos. 11131008, 61173009 and 11661131004)

\section{References}

[1] H.J. Baues, On homotopy classification problems of J.H.C. Whitehead, Lecture Notes in Math. 1172 (1985), 17-55

[2] H.J. Baues, Homotopy Type and Homology, Oxford University Press, New York, 1996

[3] H.J. Baues and Y.A. Drozd, The homotopy classification of $(n-1)$-connected $(n+4)$-dimensional polyhedra with torsion free homology, $n \geqslant 5$, Expo. Math. 17 (1999), 161-180

[4] H.J. Baues and Y.A. Drozd, Classification of stable homotopy types with torsion-free homology, Topology 40 (2011), 789-821

[5] H.J. Baues and M. Hennes, The homotopy classification of $(n-1)$-connected $(n+3)$-dimensional polyhedra, $n \geqslant 4$, Topology 30 (1991), 373-408

[6] S.C. Chang, Homology invariants and continuous mappings, Proc. Roy. Soc. London Ser. A 202 (1950), 253-263

[7] J.M. Cohen, Stable Homotopy, Springer, Berlin, Heidelberg, 1970

[8] Y.A. Drozd, On classification of torsion free polyhedra, Preprint Ser., MaxPlanck-Institut Math. 92 (2005) 
[9] J. Neisendorfer, Primary Homotopy Theory, Amer. Math. Soc., Rhode Island, 1980

[10] J. Neisendorfer, Algebraic Methods in Unstable Homotopy Theory, CUP, 2010

[11] J.Z. Pan and Z.J. Zhu, The classification of 2 and 3 torsion free polyhedra, Acta Math. Sinica (Eng. Ser.) 31.11 (2015), 1659-1682

[12] J.Z. Pan and Z.J. Zhu, The stable homotopy classification of $\mathbf{A}_{n}^{4}$-polyhedra with 2 torsion free homology, Sci. China Math. 59.6 (2016), 1141-1162

[13] R.M. Switzer, Algebraic Topology-Homology and Homotopy, Springer-Verlag, Berlin, 1975

[14] P. Selick and J. Wu, The functor $A^{\text {min }}$ on p-local spaces, Math. Z. 253.3 (2006), 435-451

[15] H.M. Unsöld, $A_{n}^{4}$-polyhedra with free homology, Manuscripta Math. 65 (1989), $123-146$

[16] J. Wu, Homotopy Theory of the Suspensions of the Projective Plane, Mem. Amer. Math. Soc., Rhode Island, 2003

[17] Z. Zhu and J.Z. Pan, The decomposability of smash product of $A_{n}^{2}$-complexes, preprint, arXiv: 1606.00624v1

[18] http://topology-octopus.herokuapp.com/problemsinhomotopytheory/show/ Unstable+homotopy+theory

Zhongjian Zhu zhuzhongjian@amss.ac.cn

School of Mathematics and Information Science, Wenzhou University, Wenzhou, Zhejiang 325035, China

Jianzhong Pan pjz@amss.ac.cn

Hua Loo-Keng Key Mathematical Laboratory, Institute of Mathematics, Academy of Mathematics and Systems Science, Chinese Academy of Sciences; University of Chinese Academy of Sciences; No. 55, Zhongguancun East Road, Haidian District, Beijing, 100190, China 